Document downloaded from:

http://hdl.handle.net/10251/125109

This paper must be cited as:

Zafar, F.; Cordero Barbero, A.; Sultana, S.; Torregrosa Sánchez, JR. (2018). Optimal iterative methods for finding multiple roots of nonlinear equations using weight functions and dynamics. Journal of Computational and Applied Mathematics. 342:352-374.

https://doi.org/10.1016/j.cam.2018.03.033

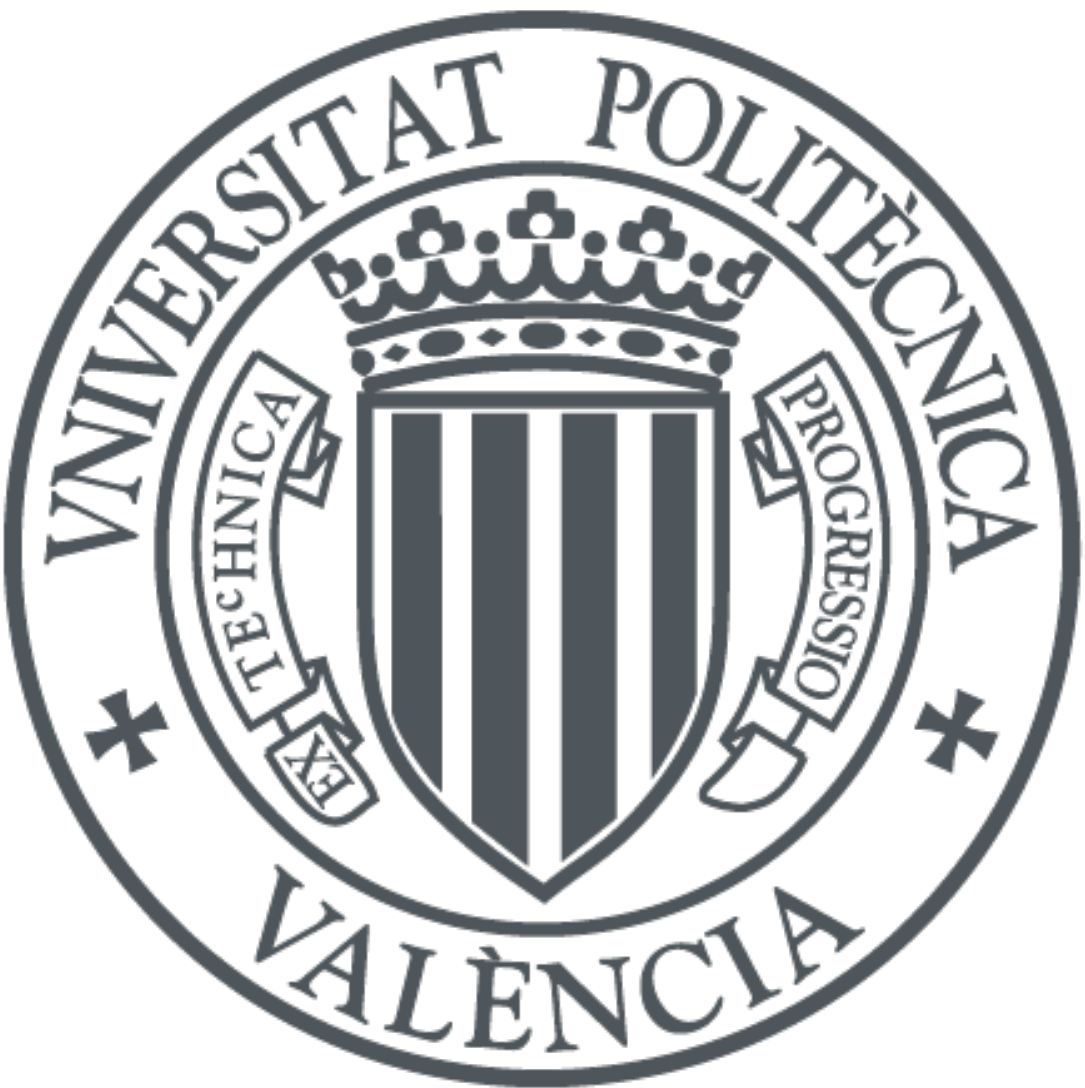

The final publication is available at

http://doi.org/10.1016/j.cam.2018.03.033

Copyright Elsevier

Additional Information 


\title{
Optimal Iterative Methods for Finding Multiple Roots of Nonlinear Equations Using Weight Functions and Dynamics is
}

\author{
Fiza Zafar ${ }^{\mathrm{a}, \mathrm{b}}$, Alicia Cordero ${ }^{\mathrm{b}}$, Sana Sultana ${ }^{\mathrm{a}}$, Juan R. Torregrosa ${ }^{\mathrm{b}}$ \\ ${ }^{a}$ Centre for Advanced Studies in Pure and Applied Mathematics, \\ Bahauddin Zakariya University, Multan 60800, Pakistan \\ ${ }^{b}$ Instituto de Matemáticas Multidisciplinar, Universitat Politècnica de València, Camino de Vera, s/n, 46022-Valencia, Spain
}

\begin{abstract}
In this paper, we propose a family of iterative methods for finding multiple roots, with known multiplicity, by means of the introduction of four univariate weight functions. With the help of these weight functions, that play an important role in the development of higher order convergent iterative techniques, we are able to construct three-point eight-order optimal multiple-root finders. Also, numerical experiments have been applied to a number of test equations for different special schemes from this family satisfying the conditions given in the convergence analysis. We have also compared the basins of attraction of some proposed and known methods in order to check the wideness of the sets of converging initial points for each problem.
\end{abstract}

Keywords: Nonlinear equations; optimal iterative methods; multiple root; basin of attraction; stability.

\section{Introduction}

Newton's method has been widely used to investigate simple or multiple zeros of a nonlinear equation. If the given function involves only a simple zero, then Newton's method converges quadratically to the exact solution provided that a proper initial guess is selected close to the exact solution. Newton's method has a drawback that it converges linearly when a given function has multiple roots. For a nonlinear equation of the form $f(x)=0$, which involves multiple roots with multiplicity $m>1$ a prior, modified Newton's method [14, 15] is given as:

$$
x_{n+1}=x_{n}-m \frac{f\left(x_{n}\right)}{f^{\prime}\left(x_{n}\right)}, \quad n=0,1,2, \ldots
$$

It efficiently locates the desired multiple zero with quadratic order convergence. The numerical scheme (1.1) is a secondorder, one-point, optimal method on the basis of Kung-Traub's conjecture [9] which states that any multipoint method without memory can reach its convergence order of at most $2^{r-1}$ for $r$ functional evaluations per iteration. In the recent past, many researchers like Li et al. [11] in (2009), Sharma and Sharma [19] and Li et al. [10] in (2010), Zhou et al. [23] in (2011), Sharifi et al. [16] in (2012), Soleymani et al. [17], Soleymani and Babajee [18], Liu and Zhou et al. [12] and Zhou et al. [24] in (2013), Thukral [20] in (2014), Behl et al. [1] and Hueso et al. [7] in (2015) and Behl et al. [2] in (2016) have presented optimal fourth-order iterative methods for multiple zeros. In addition, Li et al. [10] and Neta [13] presented optimal and non-optimal fourth-order iterative methods. Thukral [21] and Geum et al. [5, 6] have been able to present sixth order convergent methods for finding multiple roots.

\footnotetext{
This research was partially supported by Ministerio de Economía y Competitividad MTM2014-52016-C2-2-P, MTM2015-64013-P and Generalitat Valenciana PROMETEO/2016/089 and Schlumberger Foundation-Faculty for Future Program.

Email addresses: fizazafar@gmail.com(Fiza Zafar), acordero@mat.upv.es (Alicia Cordero), sanasultana8877@gmail.com (Sana Sultana), jrtorre@mat.upv.es (Juan R. Torregrosa)
} 
In 2013, Thukral [21] presented a multi-point iterative method with sixth-order convergence, which is given by

$$
\begin{aligned}
y_{n} & =x_{n}-m \frac{f\left(x_{n}\right)}{f^{\prime}\left(x_{n}\right)} \\
z_{n} & =x_{n}-m \frac{f\left(x_{n}\right)}{f^{\prime}\left(x_{n}\right)} \sum_{i=1}^{3} i\left(\frac{f\left(y_{n}\right)}{f\left(x_{n}\right)}\right)^{\frac{i}{m}}, \\
x_{n+1} & =z_{n}-m \frac{f\left(x_{n}\right)}{f^{\prime}\left(x_{n}\right)}\left(\frac{f\left(z_{n}\right)}{f\left(x_{n}\right)}\right)^{\frac{1}{m}}\left\{\sum_{i=1}^{3} i\left(\frac{f\left(y_{n}\right)}{f\left(x_{n}\right)}\right)^{\frac{i}{m}}\right\}^{2} .
\end{aligned}
$$

In 2015, Geum et al. [5], have given the following two-point, sixth-order iterative scheme, for $m>1$ :

$$
\begin{aligned}
y_{n} & =x_{n}-m \frac{f\left(x_{n}\right)}{f^{\prime}\left(x_{n}\right)}, \\
x_{n+1} & =y_{n}-Q\left(p_{n}, s_{n}\right) \frac{f\left(y_{n}\right)}{f^{\prime}\left(y_{n}\right)},
\end{aligned}
$$

where $p_{n}=\sqrt[m]{\frac{f\left(y_{n}\right)}{f\left(x_{n}\right)}}, s_{n}=\sqrt[m-1]{\frac{f^{\prime}\left(y_{n}\right)}{f^{\prime}\left(x_{n}\right)}}$ and $Q: \mathbb{C}^{2} \rightarrow \mathbb{C}$ is a holomorphic function in the neighbourhood of origin $(0,0)$.

In 2016, Geum et al. [6], have given a three-point iterative scheme with sixth-order convergence for multiple zeros involving weight function approach as follows:

$$
\begin{aligned}
y_{n} & =x_{n}-m \frac{f\left(x_{n}\right)}{f^{\prime}\left(x_{n}\right)}, m>1, \\
w_{n} & =x_{n}-m G\left(p_{n}\right) \frac{f\left(x_{n}\right)}{f^{\prime}\left(x_{n}\right)}, \\
x_{n+1} & =x_{n}-m K\left(p_{n}, t_{n}\right) \frac{f\left(x_{n}\right)}{f^{\prime}\left(x_{n}\right)},
\end{aligned}
$$

where $p_{n}=\sqrt[m]{\frac{f\left(y_{n}\right)}{f\left(x_{n}\right)}}$ and $s_{n}=\sqrt[m]{\frac{f\left(w_{n}\right)}{f\left(x_{n}\right)}}$. The weight functions $G: \mathbb{C} \rightarrow \mathbb{C}$ is analytic in a neighborhood of 0 and $K: \mathbb{C}^{2} \rightarrow \mathbb{C}$ is holomorphic in a neighborhood of $(0,0)$. All of above three schemes (1.2), (1.3), and (1.4) require four functional evaluations in order to produce sixth-order convergence with the efficiency index $6^{\frac{1}{4}}=1.5650$.

Recently, in [3] Behl et al. have developed a family optimal eighth order iterative methods given as:

$$
\begin{aligned}
y_{n} & =x_{n}-m \frac{f\left(x_{n}\right)}{f^{\prime}\left(x_{n}\right)}, m>1, \\
z_{n} & =y_{n}-u_{n} Q\left(h_{n}\right) \frac{f\left(x_{n}\right)}{f^{\prime}\left(x_{n}\right)}, \\
x_{n+1} & =z_{n}-u_{n} t_{n} G\left(h_{n}, t_{n}\right) \frac{f\left(x_{n}\right)}{f^{\prime}\left(x_{n}\right)},
\end{aligned}
$$

where $u_{n}=\sqrt[m]{\frac{f\left(y_{n}\right)}{f\left(x_{n}\right)}}, h_{n}=\frac{u_{n}}{a_{1}+a_{2} u_{n}}, t_{n}=\sqrt[m]{\frac{f\left(z_{n}\right)}{f\left(y_{n}\right)}}$ and $Q: \mathbb{C} \rightarrow \mathbb{C}$ is analytic in a neighbourhood of 0 and $G: \mathbb{C}^{2} \rightarrow \mathbb{C}$ is holomorphic in the neighbourhood of $(0,0)$.

Motivated by the need to present a family of optimal higher order convergent methods for finding multiple roots, we present a family of optimal eighth order convergence method using only four function evaluations. Section 2 provides the methodology and convergence analysis, for the proposed optimal eight-order scheme. In Section 3, some special cases of the new scheme are considered. Section 4 includes the numerical experiments and comparisons of different multiple zero finders using test functions. Finally, conclusions are given in Section 5.

\section{Construction of optimal scheme with eight-order convergence}

Let us consider the following scheme involving univariate weight functions for solving the root-finding problem: 


$$
\begin{aligned}
y_{n} & =x_{n}-m \frac{f\left(x_{n}\right)}{f^{\prime}\left(x_{n}\right)}, m>0, \\
z_{n} & =y_{n}-m u_{n} H\left(u_{n}\right) \frac{f\left(x_{n}\right)}{f^{\prime}\left(x_{n}\right)}, \\
x_{n+1} & =z_{n}-u_{n} P\left(u_{n}\right) G\left(v_{n}\right) L\left(w_{n}\right) \frac{f\left(x_{n}\right)}{f^{\prime}\left(x_{n}\right)},
\end{aligned}
$$

where the weight functions $H: \mathbb{C} \rightarrow \mathbb{C}, P: \mathbb{C} \rightarrow \mathbb{C}, G: \mathbb{C} \rightarrow \mathbb{C}$ and $L: \mathbb{C} \rightarrow \mathbb{C}$ are analytic functions in a neighborhood of 0 with $u_{n}=\left(\frac{f\left(y_{n}\right)}{f\left(x_{n}\right)}\right)^{\frac{1}{m}}, v_{n}=\left(\frac{f\left(z_{n}\right)}{f\left(y_{n}\right)}\right)^{\frac{1}{m}}$ and $w_{n}=\left(\frac{f\left(z_{n}\right)}{f\left(x_{n}\right)}\right)^{\frac{1}{m}}$. In the next Theorem 1 , we show that the order of convergence of the proposed scheme will reach at optimal eight order without using additional functional evaluations.

Theorem 1. Let us consider $x=\gamma$ (say) be a multiple zero with multiplicity $m>1$ of the involved function $f$. In addition, we assume that $f: \mathbb{C} \rightarrow \mathbb{C}$ is an analytical function in the region enclosing the multiple zero $\gamma$. The proposed scheme defined by (2.1) has optimal eight-order convergence, if the weight functions satisfy:

$$
\begin{aligned}
& H_{0}=H(0)=1, H_{1}=H^{\prime}(0)=2, H_{2}=H^{\prime \prime}(0), H_{3}=H^{\prime \prime \prime}(0) \text { are free real numbers } \\
& P_{0}=P(0), L_{0}=L(0), P_{1}=P^{\prime}(0)=2 P_{0}, P_{2}=P^{\prime \prime}(0)=P_{0}\left(2+H_{2}\right), \\
& L_{1}=L^{\prime}(0)=2 L_{0}, P_{3}=P^{\prime \prime \prime}(0)=P_{0}\left(-24+6 H_{2}+H_{3}\right) \\
& G_{0}=G(0)=0, G_{1}=G^{\prime}(0)=\frac{m}{L_{0} P_{0}}, G_{2}=G^{\prime \prime}(0)=\frac{2 m}{L_{0} P_{0}} \\
& P_{0}, L_{0} \text { are also free real numbers. }
\end{aligned}
$$

The error equation is given as:

$$
\begin{aligned}
e_{n+1}= & \frac{1}{48 m^{8}} c_{1}\left(c_{1}^{2}\left(m-H_{2}+9\right)-2 m c_{2}\right)\left[\left(14 m^{3}-G_{3} L_{0} P_{0}\left(H_{2}-9\right)^{2}\right.\right. \\
& -m^{2}\left(G_{3} L_{0} P_{0}+12 H_{2}-144\right) \\
& \left.+2 m\left(161-48 H_{2}+3 H_{2}^{2}+4 H_{3}-9 G_{3} L_{0} P_{0}+G_{3} H_{2} L_{0} P_{0}\right)\right) c_{1}^{4} \\
& -\left(4 m 12 m^{2}+G_{3}\left(H_{2}-9\right) L_{0} P_{0}-m\left(-72+6 H_{2}+G_{3} L_{0} P_{0}\right)\right) c_{1}^{2} c_{2} \\
& \left.+4 m^{2}\left(6 m-G_{3} L_{0} P_{0}\right) c_{2}^{2}+24 m^{3} c_{1} c_{3}\right] e_{n}^{8}+O\left(e_{n}^{9}\right),
\end{aligned}
$$

where $c_{k}=\frac{m !}{(m+k) !} \frac{f^{(m+k)}(\gamma)}{f^{(m)}(\gamma)}, k=1,2,3, \ldots$, and $e_{n}=x_{n}-\gamma$.

Proof. By expanding $f\left(x_{n}\right)$ and $f^{\prime}\left(x_{n}\right)$ by the Taylor's series expansion on $\gamma$, we have

$$
f\left(x_{n}\right)=\frac{f^{(m)}(\gamma)}{m !} e_{n}^{m}\left(1+c_{1} e_{n}+c_{2} e_{n}^{2}+c_{3} e_{n}^{3}+c_{4} e_{n}^{4}+c_{5} e_{n}^{5}+c_{6} e_{n}^{6}+c_{7} e_{n}^{7}+c_{8} e_{n}^{8}+O\left(e_{n}^{9}\right)\right)
$$

and

$$
\begin{aligned}
f^{\prime}\left(x_{n}\right)= & \frac{f^{(m)}(\gamma)}{m !} e_{n}^{m-1}\left(m+c_{1}(m+1) e_{n}+c_{2}(m+2) e_{n}^{2}+c_{3}(m+3) e_{n}^{3}\right. \\
& +c_{4}(m+4) e_{n}^{4}+c_{5}(m+5) e_{n}^{5}+c_{6}(m+6) e_{n}^{6}+c_{7}(m+7) e_{n}^{7} \\
& \left.+c_{8}(m+8) e_{n}^{8}+O\left(e_{n}^{9}\right)\right)
\end{aligned}
$$

respectively. By inserting the above expressions (2.3) and (2.4), in the first sub-step of our method (2.1), we obtain:

$$
y_{n}-\gamma=\frac{c_{1} e_{n}^{2}}{m}+\frac{\left(2 m c_{2}-(m+1) c_{1}^{2}\right) e_{n}^{3}}{m^{2}}+\sum_{i=0}^{4} A_{i} e_{n}^{i+4}+O\left(e_{n}^{9}\right),
$$


where $A_{i}=A_{i}\left(m, c_{1}, c_{2}, \ldots, c_{8}\right), i=0,1, \ldots$, are given in term of $m, c_{1}, c_{2}, \ldots, c_{8}$ with explicitly written two coefficients

$$
\begin{aligned}
A_{0}= & \frac{1}{m^{3}}\left(3 c_{3} m^{2}+c_{1}^{3}(m+1)^{2}-c_{1} c_{2} m(3 m+4)\right) \\
\text { and } & \begin{aligned}
A_{1}= & \frac{-1}{m^{4}}\left(c_{1}^{4}(m+1)^{3}-2 c_{2} c_{1}^{2} m\left(2 m^{2}+5 m+3\right)\right. \\
& \left.+2 c_{3} c_{1} m^{2}(2 m+3)+2 m^{2}\left(c_{2}^{2}(m+2)-2 c_{4} m\right)\right) .
\end{aligned}
\end{aligned}
$$

With the help of Taylor's series expansion and expression (2.5), we have

$$
f\left(y_{n}\right)=f^{(m)}(\gamma) e_{n}^{2 m}\left[\frac{\left(\frac{c_{1}}{m}\right)^{m}}{m !}+\frac{\left(2 m c_{2}-(m+1) c_{1}^{2}\right)\left(\frac{c_{1}}{m}\right)^{m} e_{n}}{m !}+\sum_{i=0}^{6} \bar{A}_{i} e_{n}^{k+2}+O\left(e_{n}^{9}\right)\right],
$$

where $\bar{A}_{i}=\bar{A}_{i}\left(m, c_{1}, c_{2}, \ldots, c_{8}\right), i=0,1, \ldots$, and the first two coefficients are

$$
\begin{aligned}
\bar{A}_{0}= & \frac{1}{2 m ! c_{1}^{3}}\left(\frac{c_{1}}{m}\right)^{m+1}\left[c_{1}^{4}\left(m^{3}+3 m^{2}+3 m+3\right)-2 m c_{1}^{2} c_{2}\left(2 m^{2}+3 m+2\right)\right. \\
& \left.+4 c_{2}^{2} m^{2}(m-1)+6 c_{1} c_{3} m^{2}\right]
\end{aligned}
$$

and

$$
\begin{aligned}
\bar{A}_{1}= & \frac{1}{6 m^{2} m ! c_{1}^{3}}\left(\frac{c_{1}}{m}\right)^{m}\left[6 m c_{1}^{4} c_{2}\left(m^{4}+4 m^{3}+7 m^{2}+8 m+4\right)\right. \\
& -c_{1}^{6}(1+m)^{2}\left(m^{3}+4 m^{2}+5 m+8\right)+8 m^{3} c_{2}^{3}\left(m^{2}-3 m+2\right) \\
& -6 m^{2} c_{1}^{3} c_{3}\left(3 m^{2}+4 m+3\right)+36(m-1) m^{3} c_{1} c_{2} c_{3} \\
& \left.-12 m^{3} c_{1}^{2}\left(c_{2}^{2}\left(m^{2}+m+1\right)-2 c_{4}\right)\right] .
\end{aligned}
$$

By using expressions (2.3) and (2.6), we get

$$
u_{n}=\left(\frac{f\left(y_{n}\right)}{f\left(x_{n}\right)}\right)^{\frac{1}{m}}=\frac{c_{1} e_{n}}{m}+\frac{\left(2 m c_{2}-(m+2) c_{1}^{2}\right) e_{n}^{2}}{m^{2}}+\sum_{i=0}^{4} \widetilde{A_{i}} e_{n}^{i+3}+O\left(e_{n}^{8}\right),
$$

where $\widetilde{A_{i}}=\widetilde{A_{i}}\left(m, c_{1}, c_{2}, \ldots, c_{8}\right), i=0,1, \ldots$, whose first few coefficients are

$$
\begin{aligned}
& \widetilde{A_{0}}=\frac{1}{2 m^{3}}\left\{c_{1}^{3}\left(2 m^{2}+7 m+7\right)-2 m c_{1} c_{2}(3 m+7)+6 m^{2} c_{3}\right\}, \\
& \widetilde{A_{1}}=\frac{-1}{6 m^{4}}\left[c_{1}^{4}\left(6 m^{3}+29 m^{2}+51 m+34\right)-6 m c_{1}^{2} c_{2}\left(4 m^{2}+16 m+17\right)\right. \\
& \left.+12 m^{2} c_{1} c_{3}(2 m+5)+\left(c_{2}^{2}(m+3)-2 m c_{4}\right)\right] \text {. }
\end{aligned}
$$

Now, inserting (2.5)-(2.7) in the second sub-step of scheme (2.1), where $H\left(u_{n}\right) \approx H_{0}+H_{1} u_{n}+\frac{H_{2}}{2} u_{n}^{2}+\frac{H_{3}}{6} u_{n}^{3}$, we obtain

$$
z_{n}-\gamma=\frac{\left\{c_{1}^{3}\left(m+9-H_{2}\right)-2 m c_{1} c_{2}\right\} e_{n}^{4}}{2 m^{3}} \sum_{i=0}^{4} \widehat{A}_{i} e_{n}^{i+4}+O\left(e_{n}^{9}\right),
$$

where $\widehat{A_{i}}=\widehat{A_{i}}\left(m, c_{1}, c_{2}, \ldots, c_{8}\right), i=0,1, \ldots$, are given in terms of $m, c_{1}, c_{2}, \ldots, c_{8}$, being the two first coefficients:

$$
\begin{aligned}
\widehat{A_{0}}= & \frac{-1}{6 m^{4}}\left[c_{1}^{4}\left\{7 m^{2}+84 m+H_{3}+125-3 H_{2}(3 m+7)\right\}-6 m c_{1}^{2} c_{2}\left\{4(m+7)-3 H_{2}\right\}+12 m^{2} c_{2}^{2}+12 m^{2} c_{1} c_{3}\right], \\
\widehat{A_{1}}= & \frac{1}{24 m^{5}}\left[c_{1}^{5}\left\{46 m^{3}+677 m^{2}+1850 m+1507+4 H_{3}(4 m+9)-6 H_{2}\left(12 m^{2}+53 m+59\right)\right\}\right. \\
& \left.-4 m c_{1}^{3} c_{2}\left(13 m-9 H_{2}+83\right)-168 m^{3} c_{2} c_{3}+12 m^{2} c_{1}\left(c_{2}^{2}\left(17 m-12 H_{2}+115\right)-6 m c_{4}\right)\right] .
\end{aligned}
$$


Again, using Taylor's series expansion for $f\left(z_{n}\right)$, we get

$$
\begin{aligned}
f\left(z_{n}\right)= & f^{(m)}(\gamma) e_{n}^{4 m}\left[\frac{2^{-m}\left(\frac{c_{1}^{3}\left(m+9-H_{2}\right)-2 m c_{1} c_{2}}{m^{3}}\right)^{m}}{m !}-\frac{1}{3\left(m^{3} m !\right)}\left[2^{-m} \alpha_{0}\left(\frac{c_{1}^{3}\left(m+9-H_{2}\right)-2 m c_{1} c_{2}}{m^{3}}\right)^{m-1}\right]\right. \\
& \left.+\sum_{i=0}^{2} A_{i}^{\prime} e_{n}^{i+2}+O\left(e_{n}^{5}\right)\right]
\end{aligned}
$$

where $A_{i}^{\prime}=A_{i}^{\prime}\left(m, c_{1}, c_{2}, \ldots, c_{8}\right)$ and

$$
\alpha_{0}=c_{1}^{4}\left\{7 m^{2}+84 m+H_{3}+125-3 H_{2}(3 m+7)\right\}-6 m c_{1}^{2} c_{2}\left\{4(m+7)-3 H_{2}\right\}+12 m^{2} c_{2}^{2}+12 m^{2} c_{1} c_{3} .
$$

By using (2.6) and (2.9), we get

$$
v_{n}=\left(\frac{f\left(z_{n}\right)}{f\left(y_{n}\right)}\right)^{\frac{1}{m}}=\frac{\left(c_{1}^{2}\left(m+9-H_{2}\right)-2 m c_{2}\right) e_{n}^{2}}{2 m^{2}}+\sum_{i=0}^{3} B_{i} e_{n}^{i+3}+O\left(e_{n}^{7}\right),
$$

where $B_{i}=B_{i}\left(m, c_{1}, c_{2}, \ldots, c_{8}\right)$ and the first two terms are

$$
\begin{aligned}
B_{0}= & \frac{-1}{6 m^{3}}\left[c_{1}^{3}\left\{4 m^{2}+54 m+H_{3}+98-6 H_{2}(m+3)\right\}\right. \\
& \left.-12 m c_{1} c_{2}\left(m+9-H_{2}\right)+12 m^{2} c_{3}\right], \\
B_{1}= & \frac{1}{24 m^{4}}\left[c_{1}^{4}\left(18 m^{3}+313 m^{2}+1002 m+899+4 H_{3}(3 m+8)-6 H_{2}\left(6 m^{2}+33 m+43\right)\right)\right. \\
& -12 m c_{1}^{2} c_{2}\left(6 m^{2}+87 m+2 H_{3} 167-H_{2}(10 m+33)\right) \\
& \left.+24 m^{2} c_{1} c_{3}\left(3 m-3 H_{2}+26\right)+12 m^{2}\left(c_{2}^{2}\left(3 m+35-4 H_{2}\right)-6 m c_{4}\right)\right] .
\end{aligned}
$$

By using (2.3) and (2.9), we get:

$$
w_{n}=\left(\frac{f\left(z_{n}\right)}{f\left(x_{n}\right)}\right)^{\frac{1}{m}}=\frac{1}{2 m^{3}}\left\{c_{1}^{3}\left(m-H_{2}+9\right)-2 m c_{1} c_{2}\right\} e_{n}^{3}+\sum_{i=0}^{3} \widetilde{B_{i}} e_{n}^{i+4}+O\left(e_{n}^{8}\right),
$$

where $\widetilde{B_{i}}=\widetilde{B_{i}}\left(m, c_{1}, c_{2}, \ldots, c_{8}\right)$ and first two coefficients are

$$
\begin{aligned}
& \widetilde{B_{0}}= \frac{-1}{6 m^{4}}\left[c_{1}^{4}\left(7 m^{2}+87 m+H_{3}+152-3 H_{2}(3 m+8)\right)-6 m c_{1}^{2} c_{2}\left(4 m-3 H_{2}+29\right)\right. \\
&\left.+12 m^{2} c_{2}^{2}+12 m^{2} c_{1} c_{3}\right], \\
& \widetilde{B_{1}}= \frac{1}{24 m^{5}}\left[c_{1}^{5}\left(46 m^{3}+711 m^{2}+2246 m+2061+8 H_{3}(2 m+5)-12 H_{2}\left(6 m^{2}+30 m+37\right)\right)\right. \\
&-4 m c_{1}^{3}\left(53 m^{2}+624 m+8 H_{3}+1123-9 H_{2}(7 m+20)\right)+12 m^{2} c_{1}^{2} c_{3}\left(13 m-9 H_{2}+87\right) \\
&\left.\quad-168 m^{3} c_{2} c_{3}+12 m^{2} c_{1}\left(c_{2}^{2}\left(17 m-12 H_{2}+121\right)-6 m c_{4}\right)\right] .
\end{aligned}
$$

Next, we expand weight functions $P\left(u_{n}\right), G\left(v_{n}\right)$ and $L\left(w_{n}\right)$ in the neighborhood of origin by Taylor's series expansion up to third-order terms as follows:

$$
\begin{aligned}
& P\left(u_{n}\right) \approx P_{0}+P_{1} u_{n}+P_{2} \frac{u_{n}^{2}}{2}+P_{3} \frac{u_{n}^{3}}{6}, \\
& G\left(v_{n}\right) \approx G_{0}+G_{1} v_{n}+G_{2} \frac{v_{n}^{2}}{2}+G_{3} \frac{v_{n}^{3}}{6}, \\
& L\left(w_{n}\right) \approx L_{0}+L_{1} w_{n}+L_{2} \frac{w_{n}^{2}}{2}+L_{3} \frac{w_{n}^{3}}{6} .
\end{aligned}
$$

Now by using $(2.3)-(2.11)$ in the last sub-step of suggested method $(2.1)$, we have

$$
x_{n+1}=\frac{-G_{0} L_{0} P_{0} c_{1} e_{n}^{2}}{m^{2}}+\frac{G_{0} L_{0}\left[c_{1}^{2}\left((m+3) P_{0}-P_{1}\right)-2 m P_{0} c_{2}\right] e_{n}^{3}}{m^{3}}+\sum_{i=0}^{4} \overline{B_{i}} e_{n}^{i+4}+O\left(e_{n}^{9}\right) .
$$


We can easily obtain at-least fourth-order convergence, if we put $G_{0}=0$ in $(2.12)$, so we get

$$
x_{n+1}=\frac{\left(m-G_{1} L_{0} P_{0}\right) c_{1}\left(\left(m-H_{2}+9\right) c_{1}^{2}-2 m c_{2}\right) e_{n}^{4}}{2 m^{4}}+\sum_{i=0}^{3} B_{i}^{\prime} e_{n}^{i+5}+O\left(e_{n}^{9}\right) .
$$

Again, we will put $G_{1}=\frac{m}{L_{0} P_{0}}$ in (2.13) equation for obtaining at-least fifth-order convergence:

$$
x_{n+1}=\frac{\left(2 P_{0}-P_{1}\right) c_{1}^{2}\left(\left(m-H_{2}+9\right) c_{1}^{2}-2 m c_{1}\right) e_{n}^{5}}{2 m^{4} P_{0}}+\sum_{i=0}^{2} D_{i} e_{n}^{i+6}+O\left(e_{n}^{9}\right),
$$

where $D_{i}=D_{i}\left(m, c_{1}, c_{2}, \ldots, c_{8}, G_{2}, G_{3}, L_{0}, L_{1}, L_{2}, L_{3}, P_{0}, P_{1}, P_{2}, P_{3}, H_{2}, H_{3}\right), i=0,1, \ldots$

For getting at-least sixth-order convergence, we choose $P_{1}=2 P_{0}$ and $G_{2}=\frac{2 m}{L_{0} P_{0}}$ in equation (2.14):

$$
x_{n+1}=\frac{\left(\left(H_{2}+2\right) P_{0}-P_{2}\right) c_{1}^{3}\left(c_{1}^{2}\left(m-H_{2}+9\right)-2 m c_{2}\right) e_{n}^{6}}{4 m^{5} P_{0}}+\sum_{i=0}^{1} D_{i}^{\circ} e_{n}^{i+7}+O\left(e_{n}^{9}\right),
$$

where $D_{i}^{\circ}=D_{i}^{\circ}\left(m, c_{1}, c_{2}, \ldots, c_{8}, G_{3}, L_{0}, L_{1}, L_{2}, L_{3}, P_{0}, P_{2}, P_{3}, H_{2}, H_{3}\right), i=0,1, \ldots$

For obtaining at least seventh-order of convergence, we substitute $P_{2}=P_{0}\left(H_{2}+2\right)$ and $L_{1}=2 L_{0}$ in equation (2.15) obtaining:

$$
x_{n+1}=\frac{\left(\left(H_{3}+6 H_{2}-24\right) P_{0}-P_{3}\right) c_{1}^{4}\left(\left(m+9-H_{2}\right) c_{1}^{2}-2 m c_{2}\right) e_{n}^{7}}{12 m^{6} P_{0}}+D_{i}^{\prime} e_{n}^{8}+O\left(e_{n}^{9}\right) .
$$

Finally, we will get eighth-order convergence by putting $P_{3}=P_{0}\left(H_{3}+6 H_{2}-24\right)$ in (2.16). So,

$$
\begin{aligned}
x_{n+1}= & \frac{1}{48 m^{8}} c_{1}\left(c_{1}^{2}\left(m-H_{2}+9\right)-2 m c_{2}\right)\left[\left(14 m^{3}-G_{3} L_{0} P_{0}\left(H_{2}-9\right)^{2}-m^{2}\left(G_{3} L_{0} P_{0}+12 H_{2}-144\right)\right.\right. \\
& \left.+2 m\left(161-48 H_{2}+3 H_{2}^{2}+4 H_{3}-9 G_{3} L_{0} P_{0}+G_{3} H_{2} L_{0} P_{0}\right)\right) c_{1}^{4} \\
& -4 m\left(12 m^{2}+G_{3}\left(H_{2}-9\right) L_{0} P_{0}-m\left(-72+6 H_{2}+G_{3} L_{0} P_{0}\right)\right) c_{1}^{2} c_{2} \\
& \left.+4 m^{2}\left(6 m-G_{3} L_{0} P_{0}\right) c_{2}^{2}+24 m^{3} c_{1} c_{3}\right] e_{n}^{8}+O\left(e_{n}^{9}\right) .
\end{aligned}
$$

This expression shows that our suggested scheme (2.1) reaches optimal eight-order convergence by using only four functional evaluations i.e. $f\left(x_{n}\right), f^{\prime}\left(x_{n}\right), f\left(z_{n}\right)$ and $f\left(y_{n}\right)$ per iteration. Hence this completes the proof.

\section{Some special cases of weight functions}

In this section, we discuss some special cases of our suggested method (2.1) by using different types of weight functions $P, H, G$ and $L$. These special cases are as follows:

Case 1. When all the weight functions are polynomials:

$$
\begin{aligned}
H\left(u_{n}\right) & =1+2 u_{n}+\frac{H_{2}}{2} u_{n}^{2}+\frac{H_{3}}{6} u_{n}^{3}, \\
P\left(u_{n}\right) & =P_{0}+2 P_{0} u_{n}+\left(1+\frac{H_{2}}{2}\right) P_{0} u_{n}^{2}+\left(-4+H_{2}+\frac{H_{3}}{6}\right) P_{0} u_{n}^{3}, \\
G\left(v_{n}\right) & =\frac{m}{L_{0} P_{0}} v_{n}+\frac{m}{L_{0} P_{0}} v_{n}^{2}+G_{3} \frac{v_{n}^{3}}{6} \\
L\left(w_{n}\right) & =L_{0}+2 L_{0} w_{n}+L_{2} \frac{w_{n}^{2}}{2}+L_{3} \frac{w_{n}^{3}}{6},
\end{aligned}
$$

so for $H_{2}=H_{3}=G_{3}=L_{2}=L_{3}=0$ the method (2.1) becomes:

$$
\begin{aligned}
y_{n} & =x_{n}-m \frac{f\left(x_{n}\right)}{f^{\prime}\left(x_{n}\right)} \\
z_{n} & =y_{n}-m u_{n}\left(1+2 u_{n}\right) \frac{f\left(x_{n}\right)}{f^{\prime}\left(x_{n}\right)} \\
x_{n+1} & =z_{n}-m u_{n} v_{n} \frac{f\left(x_{n}\right)}{f^{\prime}\left(x_{n}\right)}\left[\left(1+2 u_{n}+u_{n}^{2}-4 u_{n}^{3}\right)\left(1+v_{n}\right)\left(1+2 w_{n}\right)\right] .
\end{aligned}
$$


Case 2. Let us consider (3.1) for the choice of $H_{2}=H_{3}=1, L_{2}=L_{3}=G_{3}=0$ as follows:

$$
\begin{aligned}
y_{n} & =x_{n}-m \frac{f\left(x_{n}\right)}{f^{\prime}\left(x_{n}\right)}, \\
z_{n} & =y_{n}-m u_{n}\left(1+2 u_{n}+\frac{u_{n}^{2}}{2}+\frac{u_{n}^{3}}{6}\right) \frac{f\left(x_{n}\right)}{f^{\prime}\left(x_{n}\right)}, \\
x_{n+1} & =z_{n}-m u_{n} v_{n} \frac{f\left(x_{n}\right)}{f^{\prime}\left(x_{n}\right)}\left[\left(1+2 u_{n}+\frac{3}{2} u_{n}^{2}-\frac{17}{6} u_{n}^{3}\right)\left(1+v_{n}\right)\left(1+2 w_{n}\right)\right] .
\end{aligned}
$$

Case 3. When the exponential weight function is $H(u)$, polynomial and rational weight function as $P(u), G(u)$ and $L(w)$ :

$$
\begin{aligned}
H\left(u_{n}\right) & =e^{-\frac{1}{6} u_{n}\left(-16 u_{n}^{2}-H_{3} u_{n}^{2}+6 H_{2} u_{n}^{2}-3 u_{n} H_{2}+12 u_{n}-12\right)}, \\
P\left(u_{n}\right) & =\frac{1}{6} \frac{P_{0}\left(-24-36 u_{n}+6 H_{2} u_{n}+108 u_{n}^{3}-12 H_{2} u_{n}^{3}+3 H_{2}^{2} u_{n}^{3}-4 H_{3} u_{n}^{3}\right)}{-4+2 u_{n}+H_{2} u_{n}}, \\
G\left(v_{n}\right) & =\frac{m}{L_{0} P_{0}} v_{n}+\frac{m}{L_{0} P_{0}} v_{n}^{2}+G_{3} \frac{v_{n}^{3}}{6}, \\
L\left(w_{n}\right) & =-\frac{2\left(-3 L_{2}+24 L_{0}+L_{3} w_{n}-12 L_{2} w_{n}+48 L_{0} w_{n}\right) L_{0}^{2}}{6 L_{0} L_{2}-48 L_{0}^{2}-2 L_{0} L_{3} w_{n}+12 L_{0} L_{2} w_{n}-3 L_{2}^{2} w_{n}^{2}+4 L_{3} L_{0} w_{n}^{2}} .
\end{aligned}
$$

For $H_{2}=H_{3}=L_{2}=G_{3}=0, L_{0}=L_{3}=1$ our proposed method (2.1) becomes

$$
\begin{aligned}
y_{n} & =x_{n}-m \frac{f\left(x_{n}\right)}{f^{\prime}\left(x_{n}\right)}, \\
z_{n} & =y_{n}-m u_{n} e^{-\frac{1}{6} u_{n}\left(-16 u_{n}^{2}+12 u_{n}-12\right) \frac{f\left(x_{n}\right)}{f^{\prime}\left(x_{n}\right)},} \\
x_{n+1} & =z_{n}-m u_{n} v_{n} \frac{f\left(x_{n}\right)}{f^{\prime}\left(x_{n}\right)}\left[\left(\frac{\left.-2-3 u_{n}+9 u_{n}^{3}\right)}{-2+u_{n}}\right)\left(1+v_{n}\right)\left(\frac{24+49 w_{n}}{24+w_{n}-2 w_{n}^{2}}\right)\right] .
\end{aligned}
$$

Case 4. When the combination of polynomial and rational functions are used:

$$
\begin{aligned}
H\left(u_{n}\right) & =\frac{1}{6}\left(\frac{-24-48 u_{n}+6 H_{2} u_{n}-4 u_{n}^{3} H_{3}+3 u_{n}^{3} H_{2}^{2}}{-4+H_{2} u_{n}}\right), \\
P\left(u_{n}\right) & =\frac{1}{6} \frac{P_{0}\left(-24-36 u_{n}+6 H_{2} u_{n}+108 u_{n}^{3}-12 H_{2} u_{n}^{3}+3 H_{2}^{2} u_{n}^{3}-4 H_{3} u_{n}^{3}\right)}{-4+2 u_{n}+H_{2} u_{n}} \\
G\left(v_{n}\right) & =\frac{m}{L_{0} P_{0}} v_{n}+\frac{m}{L_{0} P_{0}} v_{n}^{2}+G_{3} \frac{v_{n}^{3}}{6} \\
L\left(w_{n}\right) & =-\frac{2\left(-3 L_{2}+24 L_{0}+L_{3} w_{n}-12 L_{2} w_{n}+48 L_{0} w_{n}\right) L_{0}^{2}}{6 L_{0} L_{2}-48 L_{0}^{2}-2 L_{0} L_{3} w_{n}+12 L_{0} L_{2} w_{n}-3 L_{2}^{2} w_{n}^{2}+4 L_{3} L_{0} w_{n}^{2}} .
\end{aligned}
$$

For $L_{2}=G_{3}=0, H_{2}=H_{3}=L_{0}=L_{3}=1$ the method (2.1) becomes:

$$
\begin{aligned}
y_{n} & =x_{n}-m \frac{f\left(x_{n}\right)}{f^{\prime}\left(x_{n}\right)}, \\
z_{n} & =y_{n}-m u_{n} \frac{1}{6}\left(\frac{-24-42 u_{n}-u_{n}^{3}}{-4+u_{n}}\right) \frac{f\left(x_{n}\right)}{f^{\prime}\left(x_{n}\right)}, \\
x_{n+1} & =z_{n}-m u_{n} v_{n} \frac{f\left(x_{n}\right)}{f^{\prime}\left(x_{n}\right)}\left[\frac{1}{6} \frac{\left.-24-30 u_{n}+95 u_{n}^{3}\right)}{-4+3 u_{n}}\left(1+v_{n}\right)\left(\frac{24+49 w_{n}}{24+w_{n}-2 w_{n}^{2}}\right)\right] .
\end{aligned}
$$

In a similar way, we can find a new optimal eight order convergence iterative schemes for multiple zero by simply assigning different values of $P_{0}, L_{0}, G_{3}, L_{2}, L_{3}, H_{2}, H_{3}$, or by considering a new weight function that satisfies all the conditions of Theorem 1 . 


\section{Numerical Experiments}

In this section, we show the efficiency, convergence behavior and effectiveness of our suggested scheme. For this purpose, we consider some of the special cases of our proposed schemes namely, (3.2)-(3.5), denoted by $S 1, S 2, S 3$ and $S 4$ respectively. In addition, we choose a total number of eight test problems for comparison given by Examples 1-8. Now we compare our methods with the other existing ones of order six and eight on the basis of iteration error $\left|x_{n}-\gamma\right|$, computational order of convergence $\sigma_{n}$ and residual errors in the function $\left|f\left(x_{n}\right)\right|$. We compare our proposed method with a family of two-point sixth-order method, which were presented by Guem et al. [5], out of them we choose the following:

$$
\begin{aligned}
y_{n} & =x_{n}-m \frac{f\left(x_{n}\right)}{f^{\prime}\left(x_{n}\right)}, m>1 \\
x_{n+1} & =y_{n}-m\left[1+2(m-1)\left(p_{n}-s_{n}\right)-4 p_{n} s_{n}+s_{n}^{2}\right] \frac{f\left(y_{n}\right)}{f^{\prime}\left(y_{n}\right)},
\end{aligned}
$$

where $p_{n}=\sqrt[m]{\frac{f\left(y_{n}\right)}{f\left(x_{n}\right)}}$, and $s_{n}=\sqrt[m-1]{\frac{f^{\prime}\left(y_{n}\right)}{f^{\prime}\left(x_{n}\right)}}$, named as $G M 1$.

We also compare our scheme with another non-optimal method with sixth-order convergence based on weight function approach suggested by the same author Geum et al. [6]. Among their special cases, we select the following:

$$
\begin{aligned}
y_{n} & =x_{n}-m \frac{f\left(x_{n}\right)}{f^{\prime}\left(x_{n}\right)}, m>1, \\
w_{n} & =x_{n}-m\left[1+p_{n}+2 p_{n}^{2}\right] \frac{f\left(x_{n}\right)}{f^{\prime}\left(x_{n}\right)}, \\
x_{n+1} & =x_{n}-m\left[1+p_{n}+2 p_{n}^{2}+\left(1+2 p_{n}\right) t_{n}\right] \frac{f\left(x_{n}\right)}{f^{\prime}\left(x_{n}\right)},
\end{aligned}
$$

where $p_{n}=\sqrt[m]{\frac{f\left(y_{n}\right)}{f\left(x_{n}\right)}}$ and $s_{n}=\sqrt[m]{\frac{f\left(w_{n}\right)}{f\left(x_{n}\right)}}$, denoted by GM2.

Finally, we choose a special case of the optimal eighth order method given by Behl et al. [3] for $a_{1}=a_{2}=1$ represented by $O M$ given as:

$$
\begin{aligned}
y_{n} & =x_{n}-m \frac{f\left(x_{n}\right)}{f^{\prime}\left(x_{n}\right)}, m>1, \\
z_{n} & =y_{n}-m u_{n}\left(1+2 h_{n}+3 h_{n}^{2}\right) \frac{f\left(x_{n}\right)}{f^{\prime}\left(x_{n}\right)}, \\
x_{n+1} & =z_{n}-m u_{n} t_{n} \frac{1+2 t_{n}+3 h_{n}^{2}+h_{n}\left(2+6 t_{n}+h_{n}\right)}{1+t_{n}} \frac{f\left(x_{n}\right)}{f^{\prime}\left(x_{n}\right)},
\end{aligned}
$$

where $u_{n}=\sqrt[m]{\frac{f\left(y_{n}\right)}{f\left(x_{n}\right)}}, h_{n}=\frac{u_{n}}{1+u_{n}}$ and $t_{n}=\sqrt[m]{\frac{f\left(z_{n}\right)}{f\left(y_{n}\right)}}$.

In Tables 1-16, the comparison of the iteration error $\left|x_{i}-\gamma\right|, i=1,2,3$, the absolute residual error $\left|f\left(x_{i}\right)\right|$ for $i=1,2,3$, and the computational order of convergence $\sigma_{n}$ of our methods $S 1, S 2, S 3$ and $S 4$ with GM1, GM2 and $O M$ is shown. We have done our calculations with several number of significant digits (minimum 1000 significant digits) to minimize the round of error but due to limitations we show the results up to nine decimal places only. All computations have been performed using the programming package Mathematica 9 and Maple 16, with multiple precision arithmetic. Further, in the tables $a \times 10^{( \pm b)}$ is written as $a( \pm b)$. We use the formula given by Jay [8]:

$$
\sigma_{n} \approx \frac{\log \left|f\left(x_{n+1}\right) / f\left(x_{n}\right)\right|}{\log \left|f\left(x_{n}\right) / f\left(x_{n-1}\right)\right|}
$$

in order to calculate computational order of convergence.

The dynamical behavior of the test functions is presented in Figures 1-16. The dynamical planes that appear in this section have been generated by using the routines published in [4]. We have used a mesh of $400 \times 400$ points in an appropriate region of the complex plane. We paint in orange the points whose orbit converges to the multiple root and in black whose points whose orbit converges to another thing (strange fixed points, cycles, etc.) or diverges. We work with a tolerance of $10^{-3}$ and a maximum number of 40 iterations. The multiple root is represented in the different figures by a white star. 
Example 1. Let us consider the following standard nonlinear test function:

$$
f_{1}(x)=\left[\cos \left(\frac{\pi x}{2}\right)+x^{2}-\pi\right]^{5} .
$$

The above function has a multiple zero at $\gamma \approx 2.03472489627912661035$ of multiplicity $m=5$ with initial guess $x_{0}=2.5$.

\begin{tabular}{|l|l|l|l|l|}
\hline \multicolumn{6}{|l|}{$f_{1}(x), x_{0}=2.5, m=5, \gamma \approx 2.034724896$} & \\
\hline & $\left|x_{1}-\gamma\right|$ & $\left|x_{2}-\gamma\right|$ & $\left|x_{3}-\gamma\right|$ & $\sigma_{n}$ \\
\hline$G M 1$ & $5.220762470(-4)$ & $8.422456031(-21)$ & $1.488718831(-121)$ & 5.999888304 \\
\hline$G M 2$ & $1.111141891(-3)$ & $2.533864417(-18)$ & $3.588609343(-106)$ & 5.999684855 \\
\hline$O M$ & $2.762711102(-4)$ & $1.295945853(-28)$ & $3.044163730(-223)$ & 7.999943046 \\
\hline$S 1$ & $1.228789153(-4)$ & $6.745130071(-32)$ & $5.565313341(-250)$ & 7.999977076 \\
\hline$S 2$ & $1.055907181(-4)$ & $1.450243196(-32)$ & $1.837681327(-255)$ & 7.999981851 \\
\hline$S 3$ & $1.332830873(-4)$ & $1.679366805(-31)$ & $1.068023512(-246)$ & 7.999973241 \\
\hline$S 4$ & $1.109542612(-4)$ & $2.207929169(-32)$ & $6.019291728(-254)$ & 7.999980201 \\
\hline
\end{tabular}

Table 1: Comparison in terms of iterative error $\left|x_{n}-\gamma\right|$ for $f_{1}(x)$

\begin{tabular}{|c|c|c|c|c|}
\hline \multicolumn{5}{|c|}{$f_{1}(x), x_{0}=2.5, m=5, \gamma \approx 2.034724896$} \\
\hline & $\left|f\left(x_{1}\right)\right|$ & $\left|f\left(x_{2}\right)\right|$ & $\left|f\left(x_{3}\right)\right|$ & $\overline{\sigma_{n}}$ \\
\hline GM1 & $4.810380435(-14)$ & $5.249218640(-98)$ & $9.056581698(-602)$ & 5.999888304 \\
\hline$G M 2$ & $2.103992164(-12)$ & $1.293652895(-85)$ & $7.371091759(-525)$ & 5.999684855 \\
\hline$O M$ & $1.994805140(-15)$ & $4.527257350(-137)$ & $3.237732291(-1110)$ & 7.999943046 \\
\hline$S 1$ & $3.470811790(-17)$ & $1.729229137(-153)$ & $6.612246055(-1244)$ & 7.999977076 \\
\hline$S 2$ & $1.626120684(-17)$ & $7.945204563(-157)$ & $2.595684552(-1271)$ & 7.999981851 \\
\hline S3 & $5.211125989(-17)$ & $1.654355054(-151)$ & $1.721095493(-1227)$ & 7.999973241 \\
\hline$S 4$ & $1.954088073(-17)$ & $6.498701433(-156)$ & $9.786510375(-1264)$ & 7.999980201 \\
\hline
\end{tabular}

Table 2: Comparison in terms of absolute residual errors $\left|f\left(x_{n}\right)\right|$ for $f_{1}(x)$

In Tables 1 and 2 we observe that our method has an error in the third iteration $\left|x_{3}-\gamma\right|$ or residual error $\left|f\left(x_{3}\right)\right|$ smaller than those of the known schemes. The computational order of convergence confirms the theoretical results.

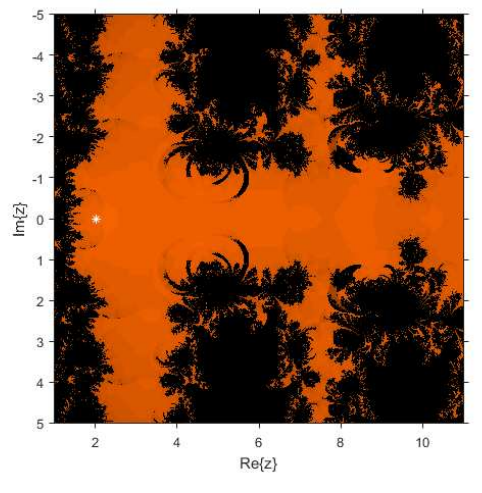

(a) GM1

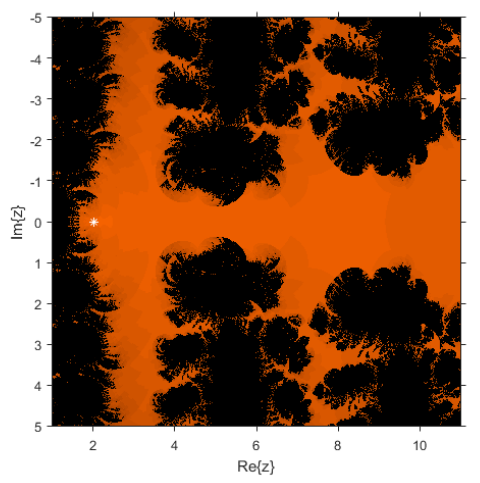

(b) GM2

Figure 1: Basins of attraction for $f_{1}(x)$ of known methods

As can be seen at Figures 1 and 2, the basins of attraction, that is, the sets of convergent initial points for each scheme are similar for all the used methods.

Example 2. We assume another standard test problem which is defined by:

$$
f_{2}(x)=\left(e^{x}+x-20\right)^{2} .
$$




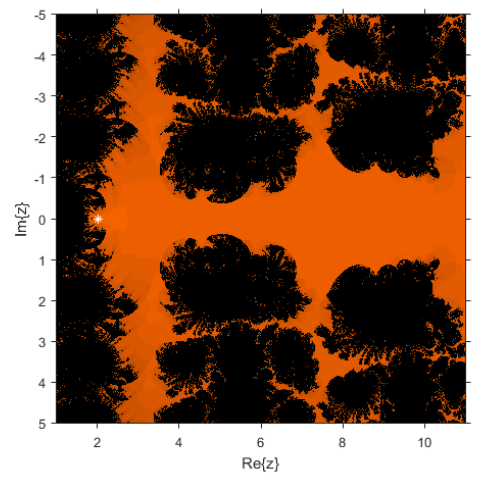

(a) $\mathrm{S} 1$

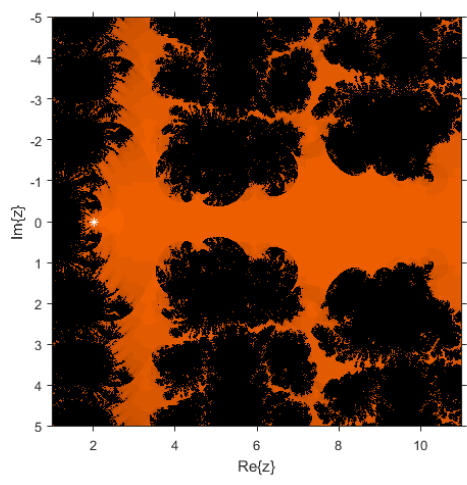

(c) S3

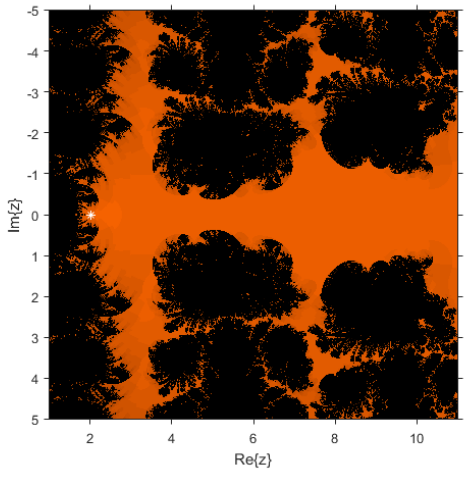

(b) S2

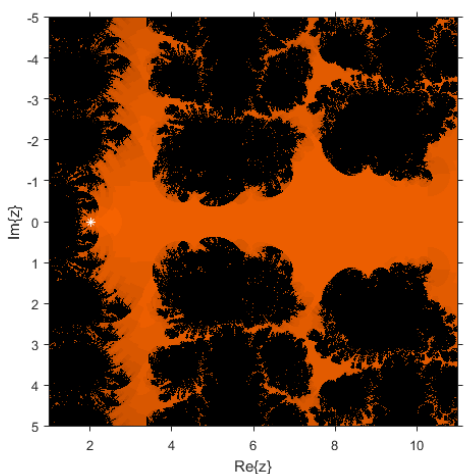

(d) S4

Figure 2: Basins of attraction for $f_{1}(x)$ of new methods

This function $f_{2}$ has multiple zero at $\gamma \approx 2.84243895378444706781$ of multiplicity $m=2$. The initial approximation is taken as $x_{0}=0.6$.

The conclusions that can be obtained from Tables 3-4 and Figures 3-4 are similar to those of the previous example.

\begin{tabular}{|l|l|l|l|l|}
\hline \multicolumn{6}{|l|}{$f_{2}(x), x_{0}=3, m=2, \gamma \approx 2.842438953$} & \\
\hline & $\left|x_{1}-\gamma\right|$ & $\left|x_{2}-\gamma\right|$ & $\left|x_{3}-\gamma\right|$ & $\sigma_{n}$ \\
\hline$G M 1$ & $1.184940430(-6)$ & $2.622019043(-37)$ & $3.078026494(-221)$ & 5.999999934 \\
\hline$G M 2$ & $5.274534652(-6)$ & $1.152582670(-32)$ & $1.254891624(-192)$ & 5.999999512 \\
\hline$O M$ & $2.076361288(-7)$ & $3.013378012(-54)$ & $5.930063701(-429)$ & 7.999999999 \\
\hline$S 1$ & $6.016268247(-8)$ & $4.258443058(-59)$ & $2.683120238(-468)$ & 7.999999997 \\
\hline$S 2$ & $4.141469194(-8)$ & $1.343043245(-60)$ & $1.642761529(-480)$ & 7.999999998 \\
\hline$S 3$ & $8.415591301(-8)$ & $9.648357994(-58)$ & $2.880074485(-457)$ & 7.999999995 \\
\hline$S 4$ & $4.900052028(-8)$ & $6.593652507(-60)$ & $7.088086766(-475)$ & 7.999999997 \\
\hline
\end{tabular}

Table 3: Comparison in terms of iterative error $\left|x_{n}-\gamma\right|$ for $f_{2}(x)$

Example 3. Let us assume another nonlinear test problem which is given below:

$$
f_{3}(x)=\left[\ln (x)+\sqrt{x^{4}+1}-2\right]^{9} .
$$

The function $f_{3}$ has a zero of multiplicity $m=9$ at $\gamma \approx 1.22281396362897310432$ and initial guess $x_{0}=10.5$.

The numerical results shown in Tables 5-6 are qualitatively similar to the previous ones and, in Figures 5-6, the basins of attraction have analogous aspects. 


\begin{tabular}{|l|l|l|l|l|}
\hline \multicolumn{5}{|c|}{$f_{2}(x), x_{0}=3, m=2, \gamma \approx 2.842438953$} \\
\hline & $\left|f\left(x_{1}\right)\right|$ & $\left|f\left(x_{2}\right)\right|$ & $\left|f\left(x_{3}\right)\right|$ & $\sigma_{n}$ \\
\hline$G M 1$ & $4.629227753(-10)$ & $2.266661714(-71)$ & $3.123631064(-439)$ & 5.999999934 \\
\hline$G M 2$ & $9.172452910(-9)$ & $4.379849588(-62)$ & $5.191913723(-382)$ & 5.999999512 \\
\hline$O M$ & $1.421415208(-11)$ & $2.993796359(-105)$ & $1.159401194(-854)$ & 7.999999999 \\
\hline$S 1$ & $1.193354388(-12)$ & $5.978837017(-115)$ & $2.373533119(-933)$ & 7.999999997 \\
\hline$S 2$ & $5.654886771(-13)$ & $5.946960028(-118)$ & $8.897419619(-958)$ & 7.999999998 \\
\hline$S 3$ & $2.334986277(-12)$ & $3.069176359(-112)$ & $2.734780442(-911)$ & 7.999999995 \\
\hline$S 4$ & $7.916193999(-13)$ & $1.433399132(-116)$ & $1.656429957(-946)$ & 7.999999997 \\
\hline
\end{tabular}

Table 4: Comparison in terms of absolute residual errors $\left|f\left(x_{n}\right)\right|$ for $f_{2}(x)$

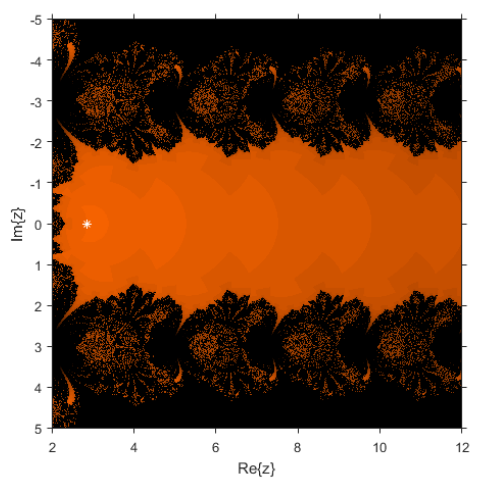

(a) GM1

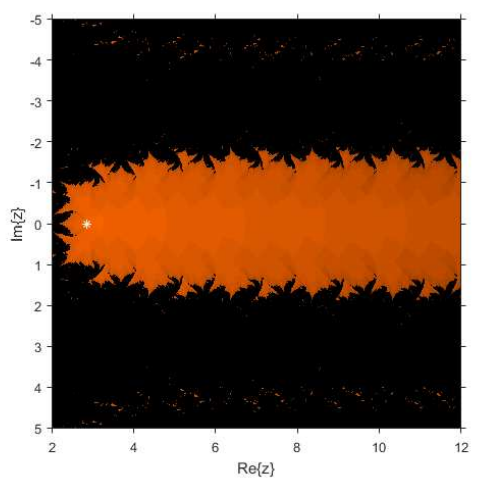

(b) GM2

Figure 3: Basins of attraction for $f_{2}(x)$ of known methods

\begin{tabular}{|l|l|l|l|l|}
\hline \multicolumn{5}{|c|}{$f_{3}(x), x_{0}=10.5, m=9, \gamma \approx 1.222813963$} \\
\hline & $\left|x_{1}-\gamma\right|$ & $\left|x_{2}-\gamma\right|$ & $\left|x_{3}-\gamma\right|$ & $\sigma_{n}$ \\
\hline$G M 1$ & 1.100433815 & $5.166891715(-3)$ & $7.860206039(-16)$ & 5.194457048 \\
\hline$G M 2$ & 1.371432030 & $1.959291353(-2)$ & $8.637659416(-12)$ & 4.655699798 \\
\hline$O M$ & 1.118274420 & $3.884821127(-3)$ & $9.873012471(-21)$ & 6.765739642 \\
\hline$S 1$ & $3.501464637(-8)$ & $7.483773054(-4)$ & $6.114496475(-27)$ & 7.205549180 \\
\hline$S 2$ & $8.787056593(-1)$ & $5.446311777(-4)$ & $3.397621553(-28)$ & 7.282937131 \\
\hline$S 3$ & $9.192937469(-1)$ & $8.477827672(-4)$ & $2.211285047(-26)$ & 7.157013052 \\
\hline$S 4$ & $8.860182249(-1)$ & $5.890154435(-4)$ & $7.334736681(-28)$ & 7.257262688 \\
\hline
\end{tabular}

Table 5: Comparison in terms of iterative error $\left|x_{n}-\gamma\right|$ for $f_{3}(x)$

\begin{tabular}{|l|l|l|l|l|}
\hline \multicolumn{5}{|c|}{$f_{3}(x), x_{0}=10.5, m=9, \gamma \approx 1.222813963$} \\
\hline & $\left|f\left(x_{1}\right)\right|$ & $\left|f\left(x_{2}\right)\right|$ & $\left|f\left(x_{3}\right)\right|$ & $\sigma_{n}$ \\
\hline$G M 1$ & $5.376098681(5)$ & $3.317195453(-17)$ & $1.423830394(-132)$ & 5.194457048 \\
\hline$G M 2$ & $6.949710765(6)$ & $5.630018548(-12)$ & $3.327496198(-96)$ & 4.655699798 \\
\hline$O M$ & $6.462280745(5)$ & $2.536717042(-18)$ & $1.108153842(-176)$ & 6.765739642 \\
\hline$S 1$ & $6.313838272(4)$ & $9.176461916(-25)$ & $1.485248686(-232)$ & 7.205549180 \\
\hline$S 2$ & $4.284891437(4)$ & $5.250854749(-26)$ & $7.501151077(-244)$ & 7.282937131 \\
\hline$S 3$ & $7.072239735(4)$ & $2.820257458(-24)$ & $1.571648600(-227)$ & 7.157013052 \\
\hline$S 4$ & $4.696527720(4)$ & $1.062940955(-25)$ & $7.638597576(-241)$ & 7.257262688 \\
\hline
\end{tabular}

Table 6: Comparison in terms of absolute residual errors $\left|f\left(x_{n}\right)\right|$ for $f_{3}(x)$

Example 4. Let us suppose another standard test function which is defined below:

$$
f_{4}(x)=(\cos x-x)^{3} .
$$

The function $f_{4}$ has a multiple zero with multiplicity $m=3$ at $\gamma \approx 0.73908513321516064165$ and initial guess $x_{0}=1$. 


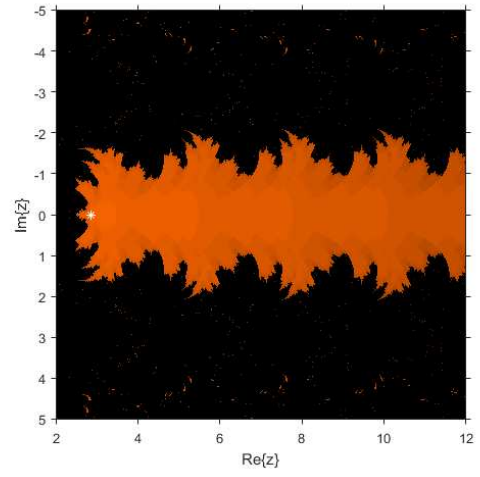

(a) $\mathrm{S} 1$

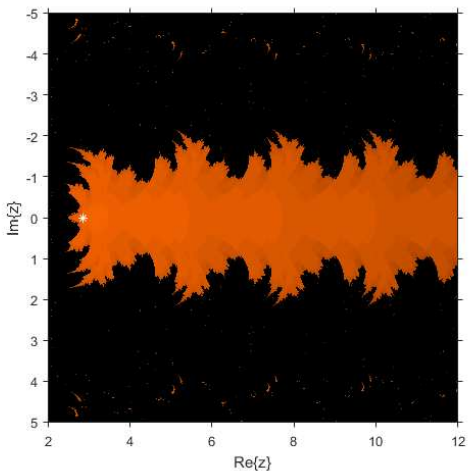

(c) $\mathrm{S} 3$

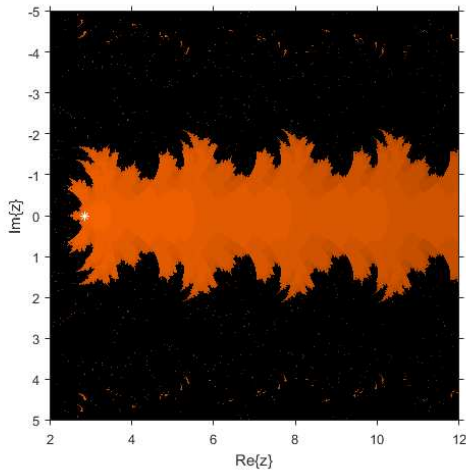

(b) S2

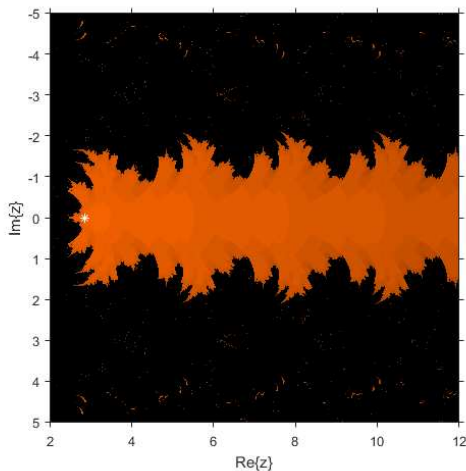

(d) S4

Figure 4: Basins of attraction for $f_{2}(x)$ of new methods

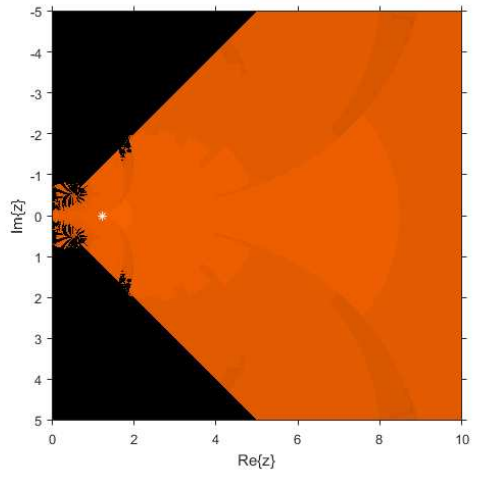

(a) GM1

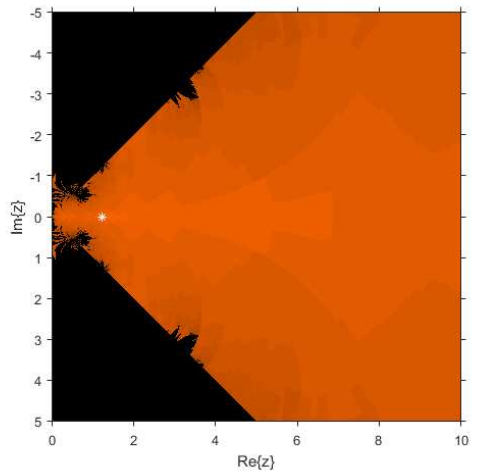

(b) GM2

Figure 5: Basins of attraction for $f_{3}(x)$ of known methods

In this example $f_{4}(x)$, the basins of attraction of methods GM1 and GM2 (see Figure 7) are wider than those corresponding to S1, S2, S3 and S4 (see Figure 8). The numerical results shown in Tables 7-8 show the good performance of the new schemes.

\section{Example 5. Let us consider}

$$
f_{5}(x)=\left(\sin ^{2} x-x^{2}+1\right)^{2} .
$$

The function $f_{5}$ has a multiple zero at $\gamma \approx 1.40449164821534122603$ with multiplicity $m=2$, and initial guess $x_{0}=2$. 


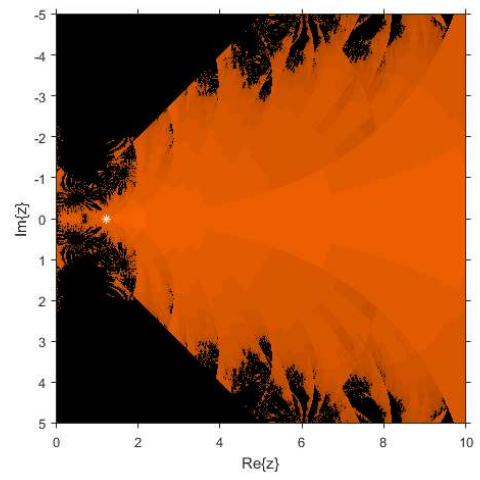

(a) $\mathrm{S} 1$

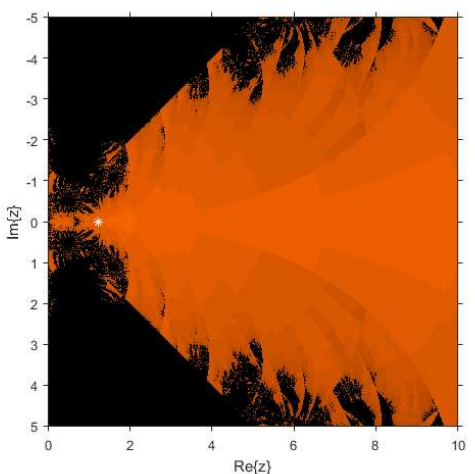

(c) $\mathrm{S} 3$

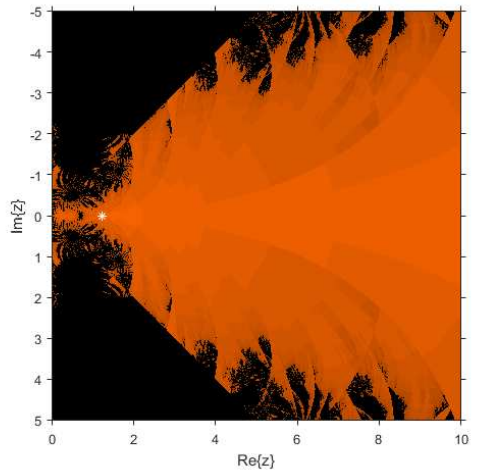

(b) $\mathrm{S} 2$

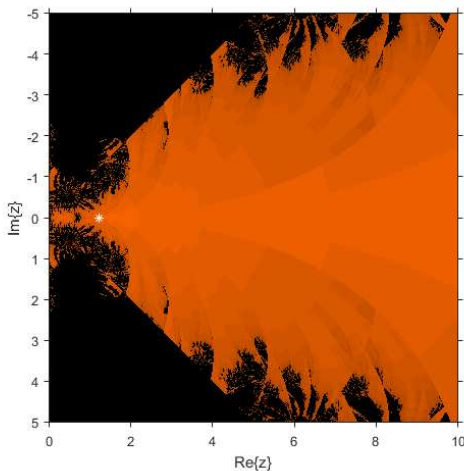

(d) S4

Figure 6: Basins of attraction for $f_{3}(x)$ of new methods

\begin{tabular}{|l|l|l|l|l|}
\hline \multicolumn{6}{|l|}{$f_{4}(x), x_{0}=1, m=3, \gamma \approx 0.739085133$} \\
\hline & $\left|x_{1}-\gamma\right|$ & $\left|x_{2}-\gamma\right|$ & $\left|x_{3}-\gamma\right|$ & $\sigma_{n}$ \\
\hline$G M 1$ & $1.143472639(-6)$ & $2.264182681(-38)$ & $1.364669639(-228)$ & 5.999999918 \\
\hline$G M 2$ & $2.553088759(-6)$ & $6.835881398(-36)$ & $2.518668790(-213)$ & 5.999999784 \\
\hline$O M$ & $8.481354395(-8)$ & $4.488396983(-59)$ & $2.761212765(-469)$ & 7.999999995 \\
\hline$S 1$ & $3.501464637(-8)$ & $1.454164026(-62)$ & $1.286834499(-497)$ & 7.999999998 \\
\hline$S 2$ & $3.007233122(-8)$ & $3.434924257(-63)$ & $9.952169910(-503)$ & 7.999999998 \\
\hline$S 3$ & $3.761173109(-8)$ & $2.969574320(-62)$ & $4.483955610(-495)$ & 7.999999998 \\
\hline$S 4$ & $3.094738237(-8)$ & $4.594985643(-63)$ & $1.085340805(-501)$ & 7.999999998 \\
\hline
\end{tabular}

Table 7: Comparison in terms of iterative error $\left|x_{n}-\gamma\right|$ for $f_{4}(x)$

\begin{tabular}{|l|l|l|l|l|}
\hline \multicolumn{6}{|l|}{$f_{4}(x), x_{0}=1, m=3, \gamma \approx 0.7390851332$} \\
\hline & $\left|f\left(x_{1}\right)\right|$ & $\left|f\left(x_{2}\right)\right|$ & $\left|f\left(x_{3}\right)\right|$ & $\sigma_{n}$ \\
\hline$G M 1$ & $7.008773523(-18)$ & $5.441250983(-113)$ & $1.191370810(-683)$ & 5.999999918 \\
\hline$G M 2$ & $7.801225670(-17)$ & $1.497434806(-105)$ & $7.489926365(-638)$ & 5.999999784 \\
\hline$O M$ & $2.859960418(-21)$ & $4.238753403(-175)$ & $9.868791727(-1406)$ & 7.999999995 \\
\hline$S 1$ & $2.012396762(-22)$ & $1.441466509(-185)$ & $9.989240126(-1491)$ & 7.999999998 \\
\hline$S 2$ & $1.274869299(-22)$ & $1.899834898(-187)$ & $4.620805750(-1506)$ & 7.999999998 \\
\hline$S 3$ & $2.494217627(-22)$ & $1.227571969(-184)$ & $4.226182817(-1483)$ & 7.999999998 \\
\hline$S 4$ & $1.389428292(-22)$ & $4.547962158(-187)$ & $5.993255053(-1503)$ & 7.999999998 \\
\hline
\end{tabular}

Table 8: Comparison in terms of absolute residual errors $\left|f\left(x_{n}\right)\right|$ for $f_{4}(x)$ 


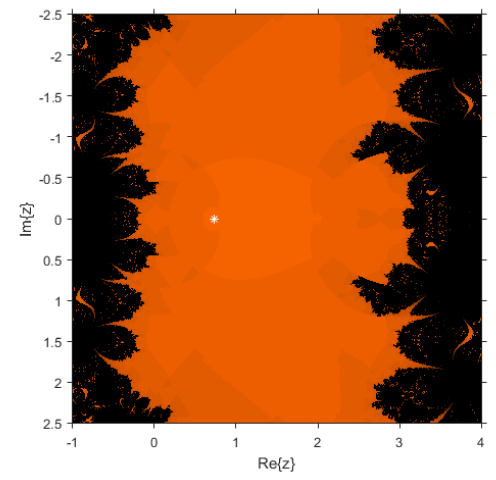

(a) GM1

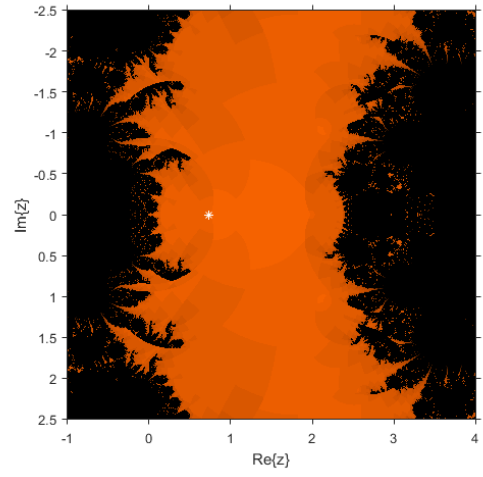

(b) GM2

Figure 7: Basins of attraction for $f_{4}(x)$ of known methods

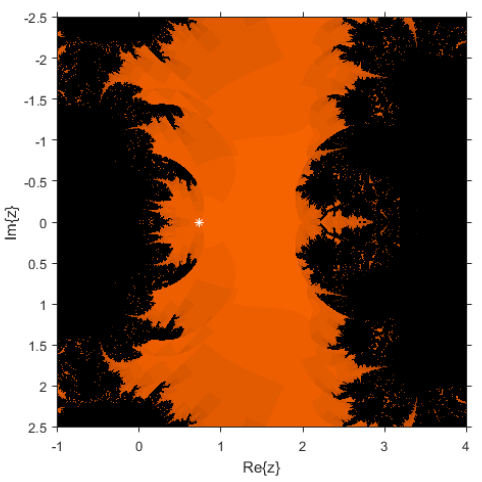

(a) $\mathrm{S} 1$

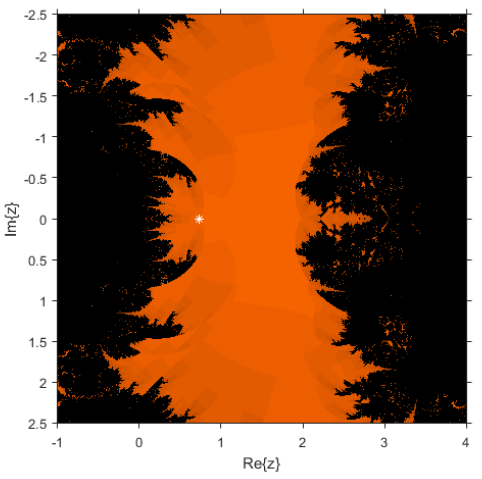

(c) $\mathrm{S} 3$

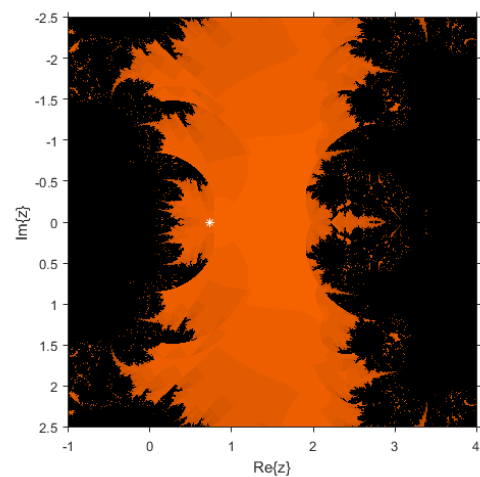

(b) S2

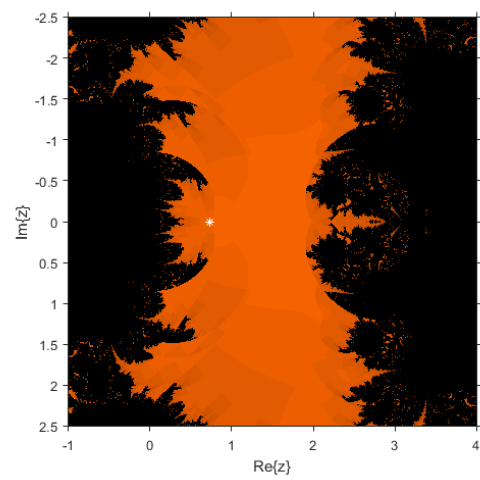

(d) S4

Figure 8: Basins of attraction for $f_{4}(x)$ of new methods

Example 6. Let us define:

$$
f_{6}(x)=\left(x^{4}-2 x^{2}+1\right)^{3} .
$$

The multiple zero of function $f_{6}, \gamma=1$, has multiplicity $m=6$ and initial guess $x_{0}=2.2$.

Example 7. Let us consider

$$
f_{7}(x)=\left(x^{3}+4 x^{2}-10\right)^{6} .
$$

The function $f_{7}$ has a zero of multiplicity $m=6$ at $\gamma \approx 1.36523001341409684576$ and initial guess $x_{0}=3$. 


\begin{tabular}{|l|l|l|l|l|}
\hline \multicolumn{5}{|l|}{$f_{5}(x), x_{0}=2, m=2, \gamma \approx 1.404491648$} \\
\hline & $\left|x_{1}-\gamma\right|$ & $\left|x_{2}-\gamma\right|$ & $\left|x_{3}-\gamma\right|$ & $\sigma_{n}$ \\
\hline$G M 1$ & $4.870872815(-3)$ & $3.238330049(-14)$ & $2.878860879(-81)$ & 5.997983222 \\
\hline$G M 2$ & $8.364077694(-3)$ & $2.686261557(-12)$ & $3.157588207(-69)$ & 5.995064976 \\
\hline$O M$ & $3.733996390(-3)$ & $1.735208010(-18)$ & $3.905151979(-144)$ & 7.998368341 \\
\hline$S 1$ & $1.989845761(-3)$ & $3.773039180(-21)$ & $6.424492009(-163)$ & 7.999233393 \\
\hline$S 2$ & $1.782676109(-3)$ & $1.110205003(-21)$ & $2.550303431(-167)$ & 7.999374198 \\
\hline$S 3$ & $2.089149870(-3)$ & $7.429195419(-21)$ & $1.942062269(-160)$ & 7.999127318 \\
\hline$S 4$ & $1.828746306(-3)$ & $1.569755717(-21)$ & $4.705271277(-166)$ & 7.999319213 \\
\hline
\end{tabular}

Table 9: Comparison in terms of iterative error $\left|x_{n}-\gamma\right|$ for $f_{5}(x)$

\begin{tabular}{|l|l|l|l|l|}
\hline \multicolumn{5}{|l|}{$f_{5}(x), x_{0}=2, m=2, \gamma \approx 1.404491648$} \\
\hline & $\left|f\left(x_{1}\right)\right|$ & $\left|f\left(x_{2}\right)\right|$ & $\left|f\left(x_{3}\right)\right|$ & $\sigma_{n}$ \\
\hline$G M 1$ & $1.473306752(-4)$ & $6.462658702(-27)$ & $5.107523315(-161)$ & 5.997983222 \\
\hline$G M 2$ & $4.368017549(-4)$ & $4.446984291(-23)$ & $6.144407130(-137)$ & 5.995064976 \\
\hline$O M$ & $8.642820503(-5)$ & $1.855547555(-35)$ & $9.398204311(-281)$ & 7.998368341 \\
\hline$S 1$ & $2.447717915(-5)$ & $8.773070744(-41)$ & $2.543586949(-324)$ & 7.999233393 \\
\hline$S 2$ & $1.963932585(-5)$ & $7.595832192(-42)$ & $4.008232718(-333)$ & 7.999374198 \\
\hline$S 3$ & $2.698542362(-5)$ & $3.401359733(-40)$ & $2.324316702(-319)$ & 7.999127318 \\
\hline$S 4$ & $2.066902234(-5)$ & $1.518564168(-41)$ & $1.364389398(-330)$ & 7.999319213 \\
\hline
\end{tabular}

Table 10: Comparison in terms of absolute residual errors $\left|f\left(x_{n}\right)\right|$ for $f_{5}(x)$

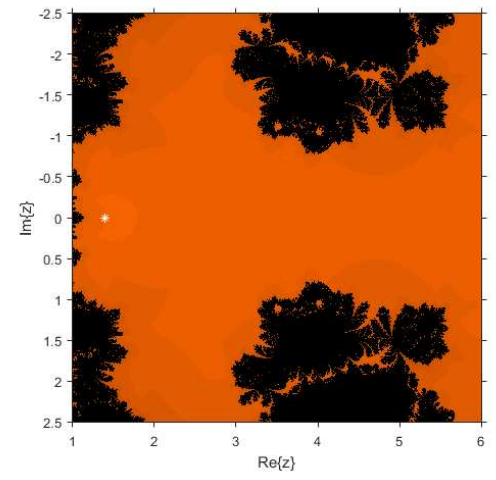

(a) GM1

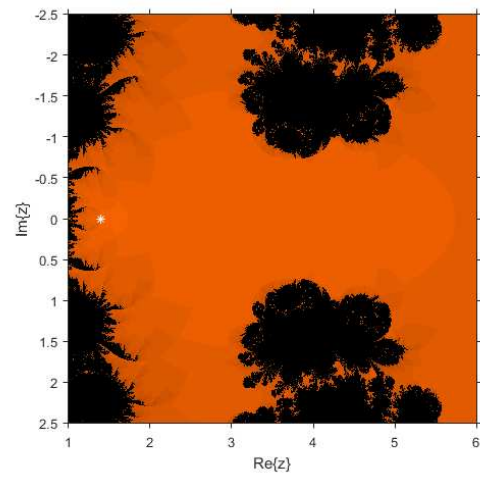

(b) GM2

Figure 9: Basins of attraction for $f_{5}(x)$ of known methods

\begin{tabular}{|l|l|l|l|l|}
\hline \multicolumn{5}{|c|}{$f_{6}(x), x_{0}=2.2, m=6, \gamma=1.0$} \\
\hline & $\left|x_{1}-\gamma\right|$ & $\left|x_{2}-\gamma\right|$ & $\left|x_{3}-\gamma\right|$ & $\sigma_{n}$ \\
\hline$G M 1$ & $1.723204281(-2)$ & $7.057617821(-12)$ & $3.614720256(-68)$ & 5.993840804 \\
\hline$G M 2$ & $2.889915685(-2)$ & $4.612259665(-10)$ & $9.025136370(-57)$ & 5.985806935 \\
\hline$O M$ & $1.509607903(-2)$ & $5.545623631(-15)$ & $2.040685202(-114)$ & 7.994270715 \\
\hline$S 1$ & $8.716435190(-3)$ & $2.448353847(-17)$ & $1.008749898(-133)$ & 7.997132194 \\
\hline$S 2$ & $7.876424462(-3)$ & $7.960208732(-18)$ & $9.115369008(-138)$ & 7.997616679 \\
\hline$S 3$ & $9.113663383(-3)$ & $4.508564491(-17)$ & $1.733962385(-131)$ & 7.996782482 \\
\hline$S 4$ & $8.077031167(-3)$ & $1.099421716(-17)$ & $1.369556332(-136)$ & 7.997436201 \\
\hline
\end{tabular}

Table 11: Comparison in terms of iterative error $\left|x_{n}-\gamma\right|$ for $f_{6}(x)$

Example 8. We define another function as:

$$
f_{8}(x)=\left(8 x e^{-x^{2}}-2 x-3\right)^{8} .
$$




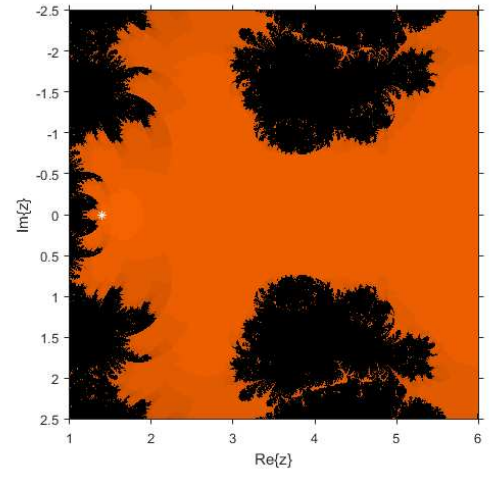

(a) $\mathrm{S} 1$

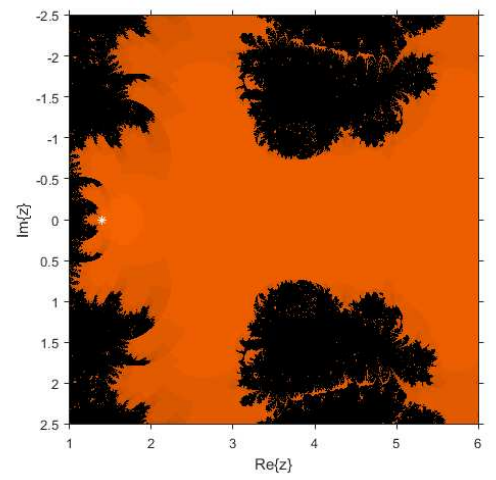

(c) $\mathrm{S} 1$

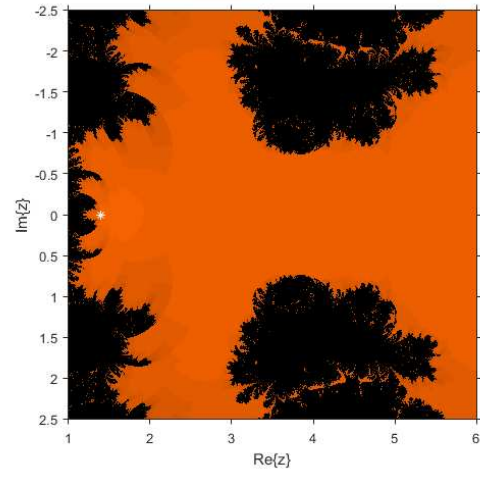

(b) S2

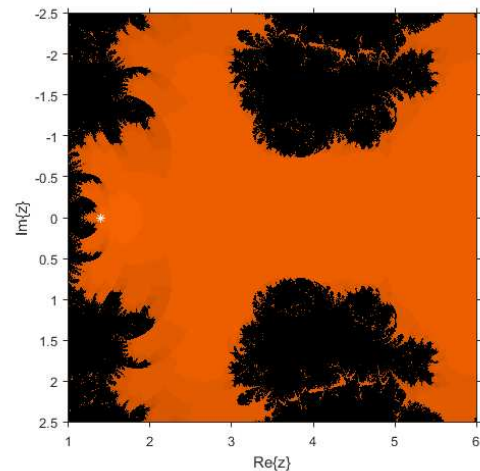

(d) S2

Figure 10: Basins of attraction for $f_{5}(x)$ of new methods

\begin{tabular}{|c|c|c|c|c|}
\hline \multicolumn{5}{|c|}{$f_{6}(x), x_{0}=2.2, m=6, \gamma=1.0$} \\
\hline & $\left|x_{1}-\gamma\right|$ & $\left|x_{2}-\gamma\right|$ & ||$x_{3}-\gamma \mid$ & $\sigma_{n}$ \\
\hline GM1 & $1.764232175(-9)$ & $7.909131501(-66)$ & $1.427670977(-403)$ & 5.993840804 \\
\hline$\overline{G M 2}$ & $4.063252417(-8)$ & $6.161159788(-55)$ & $3.458618109(-335)$ & 5.985806935 \\
\hline$O M$ & $7.924274533(-10)$ & $1.861582656(-84)$ & $4.622065179(-681)$ & 7.994270715 \\
\hline$S 1$ & $2.881013079(-11)$ & $1.378558116(-98)$ & $6.743432263(-797)$ & 7.997132194 \\
\hline$S 2$ & $1.564575613(-11)$ & $1.628271081(-101)$ & $3.671347563(-821)$ & 7.997616679 \\
\hline$S 3$ & $3.768642533(-11)$ & $5.375386437(-97)$ & $1.739474264(-783)$ & 7.996782482 \\
\hline$S 4$ & $1.820508721(-11)$ & $1.130227420(-100)$ & $4.223372379(-814)$ & 7.997436201 \\
\hline
\end{tabular}

Table 12: Comparison in terms of absolute residual errors $\left|f\left(x_{n}\right)\right|$ for $f_{6}(x)$

\begin{tabular}{|l|l|l|l|l|}
\hline \multicolumn{5}{|c|}{$f_{7}(x), x_{0}=3, m=6, \gamma \approx 1.365230013$} \\
\hline & $\left|x_{1}-\gamma\right|$ & $\left|x_{2}-\gamma\right|$ & $\left|x_{3}-\gamma\right|$ & $\sigma_{n}$ \\
\hline$G M 1$ & $6.204268880(-2)$ & $9.647881165(-9)$ & $1.711905253(-49)$ & 5.974013174 \\
\hline$G M 2$ & $1.018176714(-1)$ & $5.373771561(-7)$ & $1.861210469(-38)$ & 5.937107558 \\
\hline$O M$ & $6.625432825(-2)$ & $4.264387084(-10)$ & $1.801130693(-75)$ & 7.967277307 \\
\hline$S 1$ & $4.209676560(-2)$ & $4.184325630(-12)$ & $5.021948915(-92)$ & 7.982862600 \\
\hline$S 2$ & $3.849107043(-2)$ & $1.484407431(-12)$ & $8.773237057(-96)$ & 7.985864599 \\
\hline$S 3$ & $4.382099514(-2)$ & $7.585129608(-12)$ & $7.980285872(-90)$ & 7.980549102 \\
\hline$S 4$ & $3.951876180(-2)$ & $2.094114395(-12)$ & $1.608068505(-94)$ & 7.984566726 \\
\hline
\end{tabular}

Table 13: Comparison in terms of iterative error $\left|x_{n}-\gamma\right|$ for $f_{7}(x)$ 


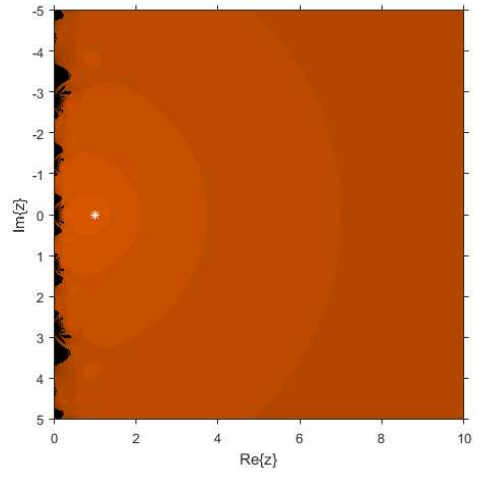

(a) GM1

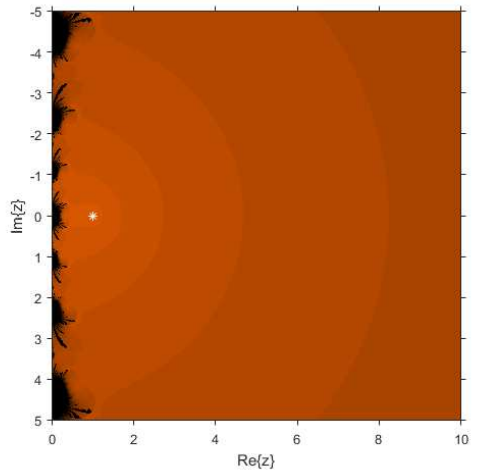

(b) GM2

Figure 11: Basins of attraction for $f_{6}(x)$ of known methods

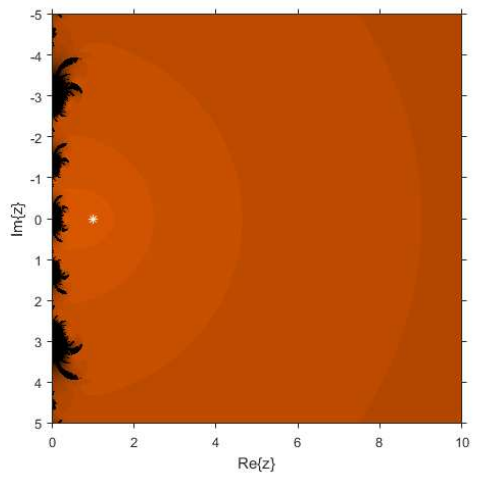

(a) $\mathrm{S} 1$

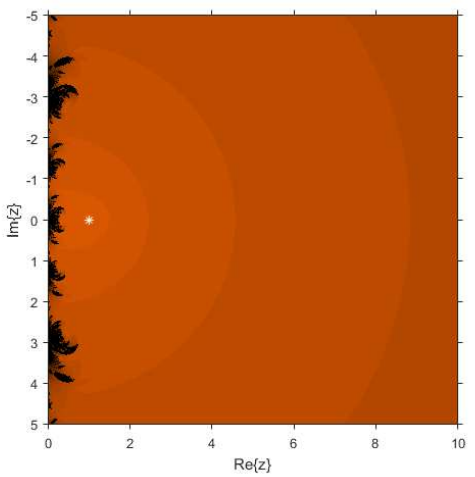

(c) S3

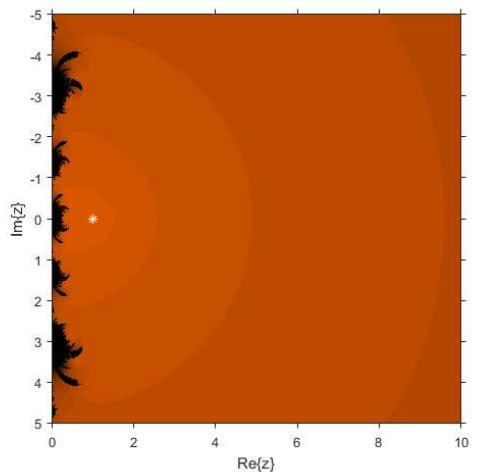

(b) S2

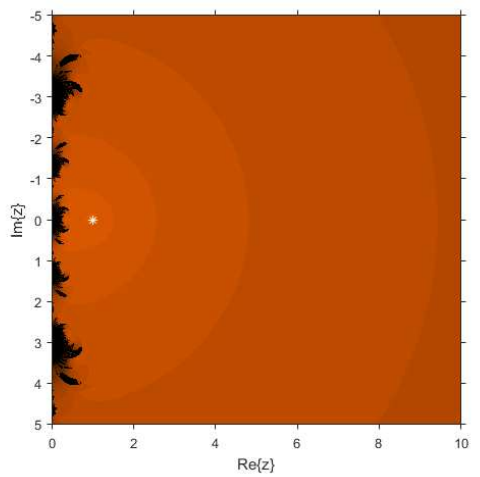

(d) S4

Figure 12: Basins of attraction for $f_{6}(x)$ of new methods

The function $f_{8}$ has a multiple zero of multiplicity $m=8$ at $\gamma \approx-1.79035317915895441218$ and initial guess $x_{0}=$ -1.2 .

Regarding Examples 5-8, it can be deduced from Tables 9-16 and Figures 9-16, that the proposed schemes show better error estimations in very similar basins of convergence. So, in the same set of initial estimations, the final results are better than those provided by existing schemes. 


\begin{tabular}{|l|l|l|l|l|}
\hline \multicolumn{6}{|l|}{$f_{7}(x), x_{0}=3, m=6, \gamma \approx 1.365230013$} & \\
\hline & $\left|f\left(x_{1}\right)\right|$ & $\left|f\left(x_{2}\right)\right|$ & $\left|f\left(x_{3}\right)\right|$ & $\sigma_{n}$ \\
\hline$G M 1$ & 1.386208354 & $1.635349358(-41)$ & $5.103841843(-286)$ & 5.974013174 \\
\hline$G M 2$ & $3.036987379(1)$ & $4.883090237(-31)$ & $8.429305217(-220)$ & 5.937107558 \\
\hline$O M$ & 2.080980548 & $1.219434775(-49)$ & $6.922934486(-442)$ & 7.967277307 \\
\hline$S 1$ & $1.276484149(-1)$ & $1.088357832(-61)$ & $3.252764362(-541)$ & 7.982862600 \\
\hline$S 2$ & $7.381106939(-2)$ & $2.169389728(-64)$ & $9.246514296(-564)$ & 7.985864599 \\
\hline$S 3$ & $1.632286362(-1)$ & $3.861863288(-60)$ & $5.237566543(-528)$ & 7.980549102 \\
\hline$S 4$ & $8.671256861(-2)$ & $1.710099080(-63)$ & $3.506276298(-556)$ & 7.984566726 \\
\hline
\end{tabular}

Table 14: Comparison in terms of absolute residual errors $\left|f\left(x_{n}\right)\right|$ for $f_{7}(x)$

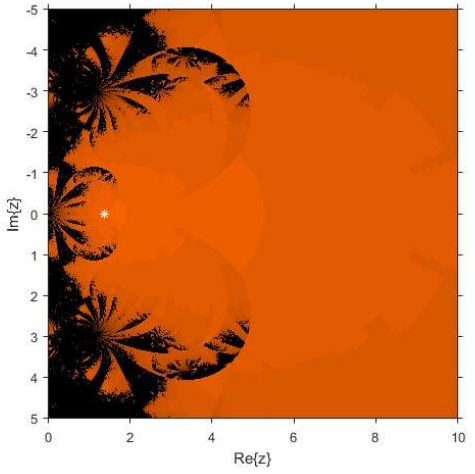

(a) GM1

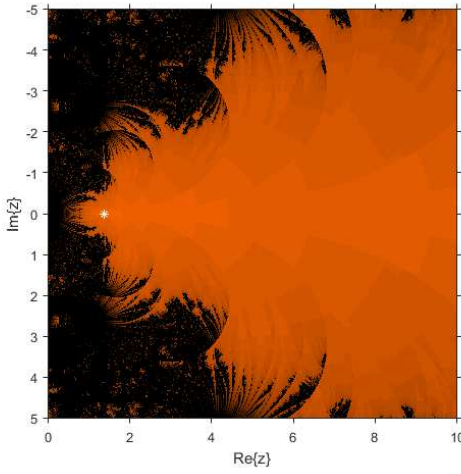

(b) GM2

Figure 13: Basins of attraction for $f_{7}(x)$ of known methods

\begin{tabular}{|l|l|l|l|l|}
\hline \multicolumn{6}{|c|}{$f_{8}(x), x_{0}=-1.2, m=8, \gamma \approx-1.790353179$} & & \\
\hline & $\left|x_{1}-\gamma\right|$ & $\left|x_{2}-\gamma\right|$ & $\left|x_{3}-\gamma\right|$ & $\sigma_{n}$ \\
\hline$G M 1$ & $4.307854679(-4)$ & $9.207188540(-22)$ & $8.779275351(-128)$ & 5.999958813 \\
\hline$G M 2$ & $2.151830489(-3)$ & $9.635795059(-17)$ & $7.819790959(-97)$ & 5.999566387 \\
\hline$O M$ & $6.590079930(-4)$ & $7.233132734(-26)$ & $1.525428460(-201)$ & 7.999918618 \\
\hline$S 1$ & $3.130404132(-4)$ & $4.981126746(-29)$ & $2.048317073(-227)$ & 7.999966699 \\
\hline$S 2$ & $2.924745644(-4)$ & $1.771835647(-29)$ & $3.214839223(-231)$ & 7.999976637 \\
\hline$S 3$ & $3.179611169(-4)$ & $8.782382901(-29)$ & $2.978315561(-225)$ & 7.999957888 \\
\hline$S 4$ & $2.946011389(-4)$ & $2.423576514(-29)$ & $5.086910238(-230)$ & 7.999969754 \\
\hline
\end{tabular}

Table 15: Comparison in terms of iterative error $\left|x_{n}-\gamma\right|$ for $f_{8}(x)$

\begin{tabular}{|l|l|l|l|l|}
\hline \multicolumn{6}{|l|}{$f_{8}(x), x_{0}=-1.2, m=8, \gamma \approx-1.790353179$} \\
\hline & $\left|f\left(x_{1}\right)\right|$ & $\left|f\left(x_{2}\right)\right|$ & $\left|f\left(x_{3}\right)\right|$ & $\sigma_{n}$ \\
\hline$G M 1$ & $4.696176340(-23)$ & $2.041187296(-164)$ & $1.394877046(-1012)$ & 5.999958813 \\
\hline$G M 2$ & $1.833474373(-17)$ & $2.937426449(-124)$ & $5.526214182(-765)$ & 5.999566387 \\
\hline$O M$ & $1.409937170(-21)$ & $2.961270251(-197)$ & $1.158780890(-1602)$ & 7.999918618 \\
\hline$S 1$ & $3.649585210(-24)$ & $1.497915470(-222)$ & $1.224740400(-1809)$ & 7.999966699 \\
\hline$S 2$ & $2.118884994(-24)$ & $3.839317649(-226)$ & $4.509627098(-1840)$ & 7.999976637 \\
\hline$S 3$ & $4.134675040(-24)$ & $1.398831837(-220)$ & $2.446990311(-1792)$ & 7.999957888 \\
\hline$S 4$ & $2.245338546(-24)$ & $4.704575558(-225)$ & $1.772144614(-1830)$ & 7.999969754 \\
\hline
\end{tabular}

Table 16: Comparison in terms of absolute residual errors $\left|f\left(x_{n}\right)\right|$ for $f_{8}(x)$

\section{Conclusion}

In this paper, we have proposed a family of iterative methods for solving nonlinear equations for multiple roots with known multiplicity. The family of schemes include univariate weight functions involving function-to-function ratio. The 


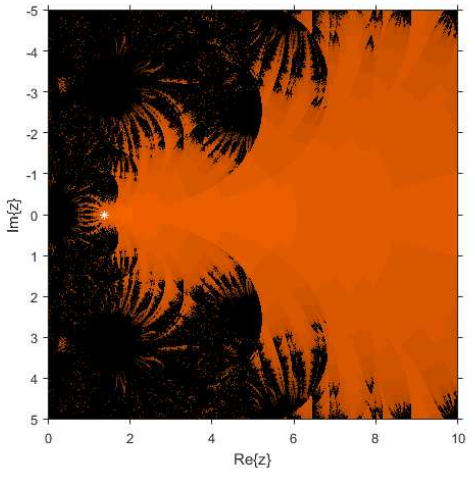

(a) $\mathrm{S} 1$

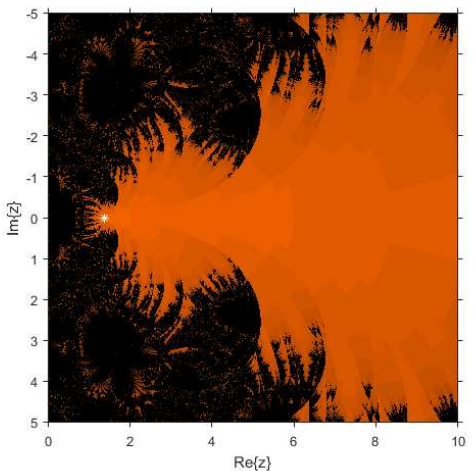

(c) $\mathrm{S} 3$

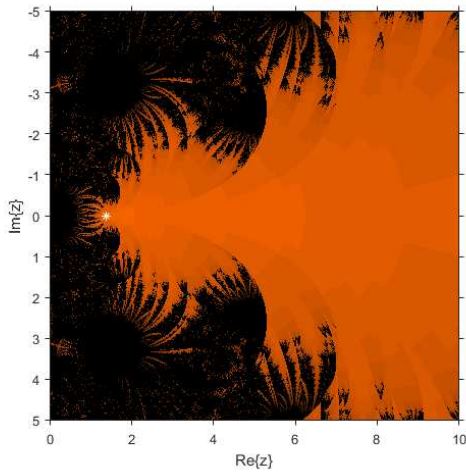

(b) S2

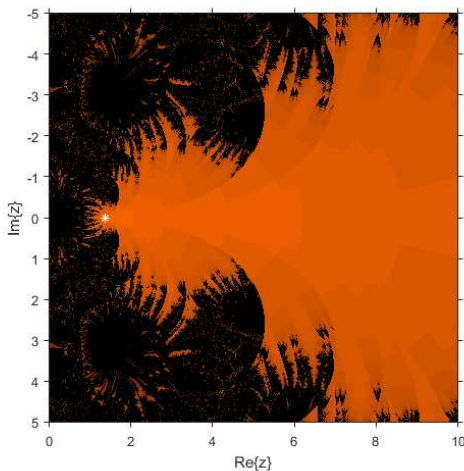

(d) S4

Figure 14: Basins of attraction for $f_{7}(x)$ of new methods

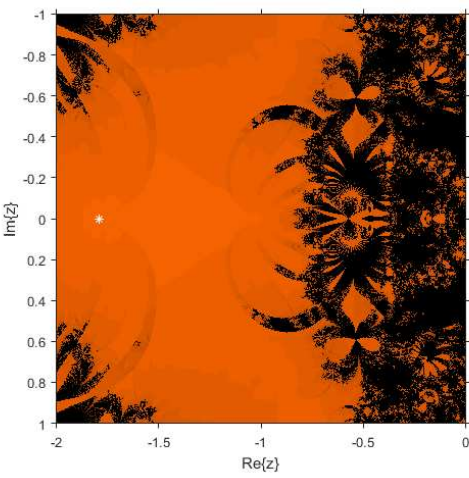

(a) GM1

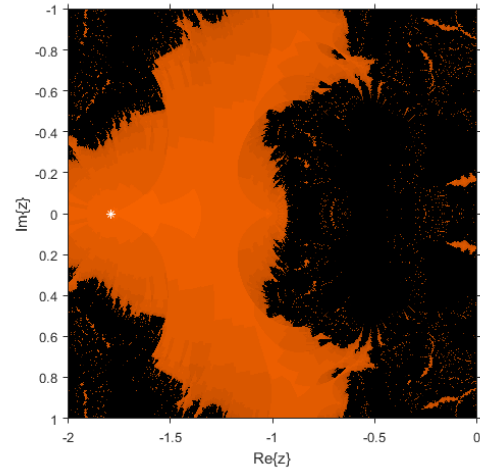

(b) GM2

Figure 15: Basins of attraction for $f_{8}(x)$ of known methods

methods involve only one derivative evaluation. The selection of weight functions yield optimal eighth order convergent methods for multiple roots. In addition, Tables 1-16 show that the proposed methods namely S1-S4 have better performance as compared to other similar known ones. The basins of attraction shown in the numerical section allow us to compare the set of initial approximations of the different methods used in the numerical section.

Acknowledgments The authors would like to thank the anonymous reviewers for their comments and suggestions that have improved the final version of the manuscript. 


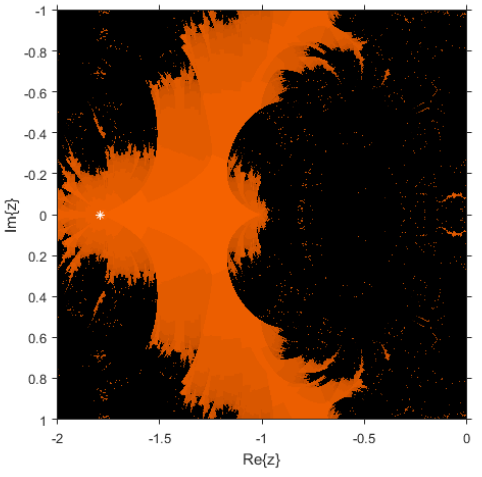

(a) $\mathrm{S} 1$

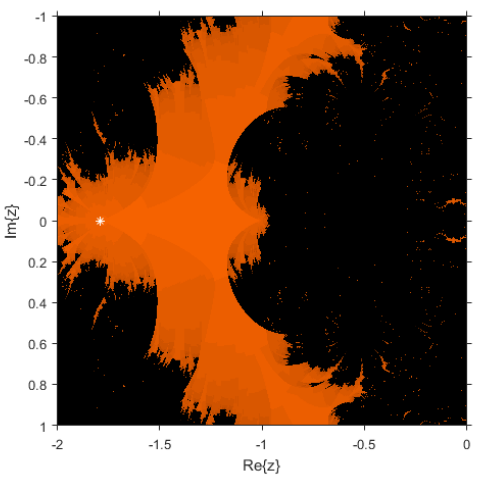

(c) $\mathrm{S} 3$

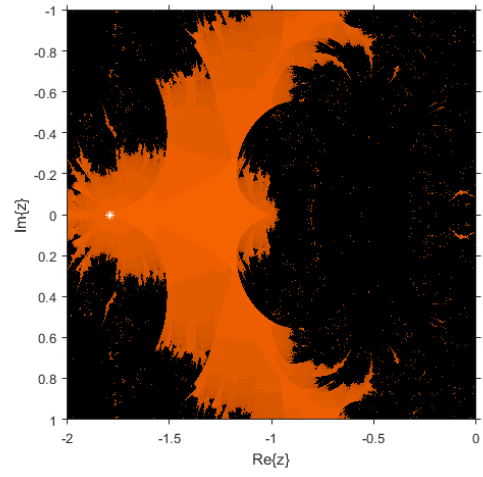

(b) S2

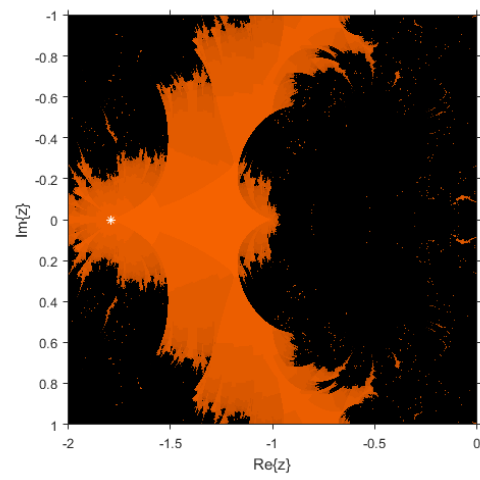

(d) S4

Figure 16: Basins of attraction for $f_{8}(x)$ of new methods

\section{References}

[1] R. Behl, A. Cordero, S.S. Motsa, J.R. Torregrosa, On developing fourth-order optimal families of methods for multiple roots and their dynamics, Appl. Math. Comput. 265(15) (2015) 520-532.

[2] R. Behl, A. Cordero, S.S. Motsa, J.R. Torregrosa, V. Kanwar, An optimal fourth-order family of methods for multiple roots and its dynamics, Numer. Algor. 71 (4) (2016) 775-796.

[3] R. Behl, A. Cordero, S.S. Motsa, J.R. Torregrosa, An eighth-order family of optimal multiple root finders and its dynamics, Numer. Algor., (2017), doi:10.1007/s11075-017-0361-6.

[4] F.I. Chicharro, A. Cordero and J. R. Torregrosa, Drawing dynamical and parameters planes of iterative families and methods, Sci. World J., 2013 (2013) ID 780153.

[5] Y.H. Geum, Y.I. Kim, B. Neta, A class of two-point sixth-order multiple-zero finders of modified double-Newton type and their dynamics, Appl. Math. Comput. 270 (2015) 387-400.

[6] Y.H. Geum, Y.I. Kim, B. Neta, A sixth-order family of three-point modified Newton-like multiple-root finders and the dynamics behind their extraneous fixed points, Appl. Math. Comput. 283 (2016) 120-140.

[7] J.L. Hueso, E. Martínez, C. Teruel, Determination of multiple roots of nonlinear equations and applications, J. Math. Chem. 53 (2015) 880-892.

[8] L.O. Jay, A note on Q-order of convergence. BIT Numer. Math. 41 (2001) 422-429.

[9] H.T. Kung, J.F. Traub, Optimal order of one-point and multipoint iteration. J. Assoc. Comput. Mach. 21 (1974) 643-651. 
[10] S.G. Li, L.Z. Cheng, B. Neta, Some fourth-order nonlinear solvers with closed formulae for multiple roots, Comput. Math. Appl. 59 (2010) 126-135.

[11] S. Li, X. Liao, L. Cheng, A new fourth-order iterative method for finding multiple roots of nonlinear equations, Appl. Math. Comput. 215 (2009) 1288-1292.

[12] B. Liu, X. Zhou, A new family of fourth-order methods for multiple roots of nonlinear equations, Non. Anal. Model. Cont. 18(2) (2013) 143-152.

[13] B. Neta, Extension of Murakami's high-order nonlinear solver to multiple roots, Int. J. Comput. Math. 87(5) (2010) 1023-1031.

[14] L.B. Rall, Convergence of Newton's process to multiple solutions, Numer. Math. 9 (1966) 23-37.

[15] E. Schröder, Überunendlichviele Algorithmenzur Auflösungder Gleichungen, Math. Annal. 2 (1870) 317-365.

[16] M. Sharifi, D.K.R. Babajee, F. Soleymani, Finding the solution of nonlinear equations by a class of optimal methods, Comput. Math. Appl. 63 (2012) 764-774.

[17] F. Soleymani, D.K.R. Babajee, T. Lofti, On a numerical technique forfinding multiple zeros and its dynamic, J. Egypt. Math. Soc. 21 (2013) 346-353.

[18] F. Soleymani, D.K.R. Babajee, Computing multiple zeros using a class of quartically convergent methods, Alex. Eng. J. 52 (2013) 531-541.

[19] J.R. Sharma, R. Sharma, Modified Jarratt method for computing multiple roots, Appl. Math. Comput. 217 (2010) $878-881$.

[20] R. Thukral, A new family of fourth-order iterative methods for solving nonlinear equations with multiple roots, J. Numer. Math. Stoch. 6 (1) (2014) 37-44.

[21] R. Thukral, Introduction to higher-order iterative methods for finding multiple roots of nonlinear equations, J. Math. 2013 (2013) Article ID 404635, 3 pages.

[22] J.F. Traub, Iterative methods for the solution of equations, Prentice-Hall, Englewood Cliffs, 1964.

[23] X. Zhou, X. Chen, Y. Song, Constructing higher-order methods for obtaining the muliplte roots of nonlinear equations, J. Comput. Math. Appl. 235 (2011) 4199-4206.

[24] X. Zhou, X. Chen, Y. Song, Families of third and fourth order methods for multiple roots of nonlinear equations, Appl. Math. Comput. 219 (2013) 6030-6038. 


\section{Answer to reviewer \#2}

Reviewer \#2: The work is interesting as it proposes an eight-order optimal scheme to find multiple roots. The proposed methods show an interesting dynamical behaviour.

In the proof of Theorem 1 it's a typo with the spacing of the left (, but I woldn't use the expression "with the help of Mathematica 9"

After equation 2.5 there is a typo "Idots" twice

After equation 3.5 "a" is missing

In a similar way, we can find "A" new optimal eight

or by considering "A" new weight function that satisf"IES"

In the new version, we have corrected all these typos.

In the beginning of section 4 . What is a vigorous method?

We have deleted this adjective.

It's desirable to be more precise with the number of digits used after equation 4.4 "We have done our calculations with several number of significant digits (minimum 1000 significant digits)"

Maybe it would be better to say it converges to something different from the root instead of another thing in the last paragraph of page 8.

After Table 2 it should be confirmS

The way in which Tables, dynamical planes and the text appears after example 3 is a little bit confusing.

References appear mixed up with dynamical planes

The conclusion is too brief in my opinion

In the corrected version, we have taken into account all these suggestions.

Thank you very much for your comments and suggestions that have improved this manuscript.

The authors 